\title{
Hydrodynamic excitations in hot QCD plasma
}

\author{
Navid Abbasi, ${ }^{1}$ Davood Allahbakhshi, ${ }^{1}$ Ali Davody, ${ }^{2}$ and Seyed Farid Taghavi ${ }^{3}$ \\ ${ }^{1}$ School of Particles and Accelerators, Institute for Research in Fundamental Sciences (IPM), \\ P.O. Box 19395-5531, Tehran, Iran \\ ${ }^{2}$ Institute of Theoretical Physics, Regensburg University, 93040 Regensburg, Germany \\ ${ }^{3}$ School of Particles and Accelerators, Institute for Research in Fundamental Sciences (IPM), \\ P.O. Box 19395-5531, Tehran, Iran
}

(Received 4 July 2017; published 7 December 2017)

\begin{abstract}
We study the long wavelength excitations in rotating QCD fluid in the presence of an external magnetic field at finite vector and axial charge densities. We consider the fluctuations of vector and axial charge currents coupled to energy and momentum fluctuations and compute the $S O(3)$ covariant dispersion relations of the six corresponding hydrodynamic modes. Among them, there are always two scalar chiralmagnetic-vortical-heat (CMVH) waves; in the absence of a magnetic field (vorticity) these waves reduce to chiral-vortical-heat $(\mathrm{CVH})$ [chiral-magnetic-heat $(\mathrm{CMH})$ ] waves. While CMVH waves are a mixture of $\mathrm{CMH}$ and $\mathrm{CVH}$ waves, they have generally different velocities compared to the sum of velocities of the latter waves. The other four modes, which are made out of scalar-vector fluctuations, are mixed soundAlfvén waves. We show that when the magnetic field is parallel with the vorticity, these four modes are the two ordinary sound modes together with two chiral Alfvén waves propagating along the common direction of the magnetic field and vorticity.
\end{abstract}

DOI: $10.1103 /$ PhysRevD.96.126002

\section{INTRODUCTION}

The phenomenon of chiral (or anomaly-induced) transport was firstly studied in fermionic systems either weakly coupled to the electromagnetic field [1] or in the presence of rotation in the system [2]. Due to the presence of anomalies in the chiral system, macroscopic currents may be produced along the external magnetic field [chiral magnetic effect (CME)] or along the vorticity of the system [chiral vortical effect (CVE)]. The appearance of such currents is the main feature of the chiral transport theory which has been extensively studied in the literature. For example, in the context of kinetic theory, a chiral theory has been derived from the underlying quantum field theory $[3,4]$, in which the Berry monopole is responsible for the CME and CVE [4,5]. The chiral magnetic effect has been also studied numerically via lattice field theory [6-9].

In another direction, after the fluid-gravity duality showed the possibility of the presence of the missed vorticity term $[10,11]$, the issue of chiral transport was taken under study in hydrodynamics. At first sight, the parity breaking terms like the magnetic field and the vorticity seem to be in contradiction with the existence of a positive divergence entropy current in fluid dynamics; however, the necessity of the second law of thermodynamics makes a relation between these terms of the hydrodynamic currents with the underlying quantum anomalies [12].

In contrast to the coefficients of the dissipative transport, the coefficients of the parity odd terms may be entirely fixed in terms of both the anomaly coefficients and the thermodynamic variables. The anomaly-induced transport is in fact a nondissipative phenomenon and so the associated coefficients are referred to as the so-called non-dissipative transport coefficients [13]. This kind of hydrodynamic transport has been used to effectively describe different phenomena in physics, e.g., in neutron stars or supernovae in astrophysics [14-16], in the study of the origin of the magnetic fields in cosmology [17] and in the propagation of Helicons in Weyl semimetals in condensed matter physics [18]. (See also $[19,20]$.)

It has been argued that in a hot plasma of chiral fermions, e.g., the plasma of quarks and gluons produced in heavy ion collisions, the combination of the chiral separation effect (CSE) [21] and CME [22] gives rise to the propagation of a new kind of gapless excitation through the hot plasma; it is called the chiral magnetic wave (CMW) [23]. CMWs have been exploited to predict the charge asymmetries in the final state of a heavy ion collision [24]. Consistent with the prediction of chiral transport, the charge asymmetries have been actually detected in experiments at RHIC and LHC $[25,26]$. Similar predictions have been made for the propagation of chiral vortical wave $(\mathrm{CVW})$ in heavy ion plasma in [27].

The CMW found in [23] has been computed by considering the fluctuations of vector and axial charge densities, keeping the local energy and local momentum in the plasma fixed. The same result has been found in the context of chiral kinetic theory in [28]. As discussed in [28], the assumption of getting the charge fluctuations decoupled from the energy-momentum fluctuations might be justifiable in a high-temperature low-density regime or even in a high-density low-temperature regime for large $N$ theories. In this paper we compute the spectrum of the 
hydrodynamic excitations in the most general case in which the vector and axial charges fluctuations are coupled to fluctuations of energy and momentum, and we compute the corresponding spectrum of the hydrodynamic excitations in the magnetic field. We find the full spectrum of hydrodynamic modes in the system, including six different waves. As a result, in addition to the two scalar chiral magnetic-heat waves (CMHWs), we find another four collective excitations, each of them being a coherent perturbation of all six hydrodynamic variables. We will show that these four modes are mixed sound-Alfvén waves. In the direction of the magnetic field, the latter four modes are identified with two ordinary sound waves together with two chiral Alfvén waves (CAWs). The propagation of CAWs was first predicted theoretically in a chiral fluid with a single chirality $[29,30]$ in the Landau-Lifshitz frame. It has been shown that the linear fluctuations of the vorticity may couple to the magnetic field and produce a wave of momentum perturbations propagating parallel to the magnetic field. This wave, namely CAW, might even propagate at zero density in the single chirality fluid. We will show that for the propagation of the CAW in QCD fluid, both the vector and axial chemical potentials are needed to be nonzero.

We also repeat the above computations for the case of a rotating QCD type hot plasma. As a result we find two scalar chiral-vortical-heat waves (CVHWs) together with four mixed Sound-Coriolis waves. In the direction of vorticity, the latter four modes are identified with two ordinary sound waves together with two rigid rotation modes.

As a main part of the paper, we compute the hydrodynamic excitations in a rotating hot plasma which is simultaneously coupled to an external magnetic field. In this case the CMHW and CVHW mix with each other and make chiral-magneticvortical-heat waves (CMVHWs). It has been shown that in a fluid with turned off momentum perturbations the velocity of mixed waves might be equal to the sum of the velocities of individual waves when the magnetic field is parallel to the vorticity $[31,32]$. However, we show that when taking into account the momentum perturbations, even for the vorticity being along the direction of the magnetic field, the velocity of mixed waves is not in general equal to the sum of the velocities of the CMHW and CVHW.

Let us emphasize that all of our results in the paper are $S O(3)$ covariant. This means that not only propagation of hydrodynamic modes whether parallel or perpendicular to the magnetic filed and vorticity are studied here, but also our results are able to explain the propagation of waves in every other arbitrary direction with respect to the magnetic field and the rotation axis.

The paper has been organized as follows. We begin with a brief review of the hot chiral QCD plasma in Sec. II. The content of Secs. III and IV is related to detailed computations of magnetic field and vorticity, respectively. In Sec. V we consider the general case wherein both the magnetic field and the vorticity are present. In Sec. VI, we apply our theoretical results to the case of quark-gluon plasma (QGP). In the same section, we study the effect of dissipation on the hydrodynamic modes. We end with a conclusion and mention some follow-up questions in Sec. VII.

\section{HYDRODYNAMICS OF A QCD-TYPE FLUID}

We consider the QCD matter in an external magnetic field. In addition to the usual electric charge (with vector current $J^{\mu}=\bar{\psi} \gamma^{\mu} \psi$ ), this matter carries a chiral charge (with chiral current $J_{5}^{\mu}=\bar{\psi} \gamma^{\mu} \gamma^{5} \psi$ ). In the presence of a background gauge field $A_{\mu}$, the dynamical equations for this hot matter are nothing but the following conservation equations:

$$
\begin{aligned}
\partial_{\mu} T^{\mu \nu} & =F^{\nu \lambda} J_{\lambda} \\
\partial_{\mu} J^{\mu} & =0 \\
\partial_{\mu} J_{5}^{\mu} & =\mathcal{C} E_{\mu} B^{\mu},
\end{aligned}
$$

where $J^{\mu}=J_{R}^{\mu}+J_{L}^{\mu}$ and $J_{5}^{\mu}=J_{R}^{\mu}-J_{L}^{\mu}$ and $\mathcal{C}$ is the chiral anomaly coefficient. In the long-wavelength regime, when the matter is in a local equilibrium state, the energy momentum tensor $T^{\mu \nu}$, vector current $J^{\mu}$, and the chiral current $J_{5}^{\mu}$ may all be effectively expressed in terms of six degrees of freedom: three components of the flowing matter velocity $u^{\mu}$, energy density $\epsilon$, vector charge density $n$, and axial charge density $n_{5}$. We may also define the electric and magnetic fields in the rest frame of this fluid as $B^{\mu}=$ $\frac{1}{2} \epsilon^{\mu \nu \alpha \beta} u_{\nu} F_{\alpha \beta}$ and $E^{\mu}=F^{\mu \nu} u_{\nu}$, respectively [12]. For small deviations from the local equilibrium state, each of the constitutive relations of the fluid may be given in a derivative expansion as follows:

$$
\begin{aligned}
T^{\mu \nu} & =(\epsilon+p) u^{\mu} u^{\nu}+p \eta^{\mu \nu}+\tau^{\mu \nu} \\
J^{\mu} & =n u^{\mu}+\nu^{\mu} \\
J_{5}^{\mu} & =n_{5} u^{\mu}+\nu_{5}^{\mu},
\end{aligned}
$$

with $\tau^{\mu \nu}, \nu^{\mu}$, and $\nu_{5}^{\mu}$ as the derivative corrections to fluid currents. In the Landau-Lifshitz frame where $u_{\mu} \tau^{\mu \nu}=0$, $u_{\mu} \nu^{\mu}=0$, and $u_{\mu} \nu_{5}^{\mu}=0$ [33], up to first order in derivative expansion we have

$$
\begin{gathered}
\tau^{\mu \nu}=-\eta P^{\mu \alpha} P^{\nu \beta}\left(\partial_{\alpha} u_{\beta}+\partial_{\beta} u_{\alpha}\right)-\left(\zeta-\frac{2}{3} \eta\right) P^{\mu \nu} \partial . u \\
\nu^{\mu}=-\sigma T P^{\mu \nu} \partial_{\nu}\left(\frac{\mu}{T}\right)+\sigma E^{\mu}+\xi \omega^{\mu}+\xi_{B} B^{\mu} \\
\nu_{5}^{\mu}=-\sigma_{5} T P^{\mu \nu} \partial_{\nu}\left(\frac{\mu}{T}\right)+\sigma_{5} E^{\mu}+\xi_{5} \omega^{\mu}+\xi_{B 5} B^{\mu}
\end{gathered}
$$

with the vorticity defining as $\omega^{\mu}=\frac{1}{2} \epsilon^{\mu \nu \alpha \beta} u_{\nu} \partial_{\alpha} u_{\beta}$. The coefficients $\eta, \zeta, \sigma$, and $\sigma_{5}$ are dissipative transport coefficients. In the following we mainly study the nondissipative 
fluids. Thus, the only relevant coefficients are the anomalous transport coefficients $\xi$ and $\xi_{B}$ corresponding to CVE and CME [12,34-38],

$$
\begin{aligned}
\xi & =2 \mathcal{C}\left(\mu \mu_{5}-\frac{n \mu_{5}}{3 w}\left(3 \mu^{2}+\mu_{5}^{2}\right)\right)-2 \mathcal{D} \frac{n \mu_{5}}{w} T^{2} \\
\xi_{5} & =\mathcal{C}\left(\mu^{2}+\mu_{5}^{2}-\frac{2 n_{5} \mu_{5}}{3 w}\left(3 \mu^{2}+\mu_{5}^{2}\right)\right)+\mathcal{D}\left(1-\frac{2 n_{5} \mu_{5}}{w}\right) T^{2} \\
\xi_{B} & =\mathcal{C} \mu_{5}\left(1-\frac{n \mu}{w}\right) \\
\xi_{5 B} & =\mathcal{C} \mu\left(1-\frac{n_{5} \mu_{5}}{w}\right)
\end{aligned}
$$

where the corresponding chiral anomaly and gravitational anomaly coefficients are

$$
\mathcal{C}=\frac{1}{2 \pi^{2}}, \quad \mathcal{D}=\frac{1}{6} .
$$

Hydrodynamic excitations are low-energy long-wavelength excitations around the equilibrium state in fluid. To find their dispersion relations, one has to firstly choose a set of hydrodynamic variables and then linearize the equations of motion in terms of their fluctuations around a thermodynamic solution. It is conventional to consider the microscopic conserved quantities and choose the hydrodynamic variables associatively, such as

$$
\phi_{a}=\left(\epsilon, \boldsymbol{\pi}_{i}, n, n_{5}\right), \quad a=1,2, \ldots, 6
$$

where $\epsilon, \boldsymbol{\pi}$, and $n$ have microscopic definitions given by $T^{00}(x), T^{0 i}(x)$, and $J^{0}(x)[39,40]$. However, we prefer to choose $\phi_{a}$ as it follows:

$$
\phi_{a}=\left(T, \boldsymbol{\pi}_{i}, \mu, \mu_{5}\right), \quad a=1,2, \ldots, 6
$$

where $\pi_{i}=w v_{i}$ and $w=\epsilon+p$ is the enthalpy density. This special choice makes the computations simpler when finding the hydrodynamic modes for a fluid with a general equation of state. However, the QCD equation of state obtained from the lattice calculations shows that, at high enough temperatures, QCD plasma is thermodynamically conformal. The nonconformality of QCD becomes serious at and just above the critical temperature $T_{c}$ [41]. Therefore, the results found by using the conformality assumptions are more quantitatively reliable when applied to data of LHC than when applied to those of RHIC. In this paper, we focus on QCD plasma at high enough temperatures with the following equation of state:

$$
\epsilon=\frac{1}{c_{s}^{2}} p, \quad\left(c_{s}=\frac{1}{\sqrt{3}}\right) .
$$

The thermodynamic solution in our system is given by [42]

$$
\begin{aligned}
u^{\mu} & =(1, \boldsymbol{\Omega} \times \boldsymbol{x}) \quad \Omega r \ll 1, \\
T & =\text { Const }, \quad \mu=\text { Const }, \quad \mu_{5}=\text { Const. } \\
\boldsymbol{B} & =\text { Const },
\end{aligned}
$$

with $r$ being the distance from the rotation axis. The pressure $p=p\left(T, \mu, \mu_{5}\right)$ satisfies

$$
d p=s d T+n d \mu+n_{5} d \mu_{5} .
$$

In this paper, we compute six hydrodynamic modes associated with six hydrodynamic variables in three different cases. Except in a subsection related to QCD fluid in quarkgluon-plasma experiments, we always neglect the effect of dissipation in our study. We first consider hydrodynamic excitations in the equilibrium of the QCD fluid coupled to an external magnetic field $(\boldsymbol{B} \neq 0, \boldsymbol{\Omega}=0)$. We then turn off the magnetic field and consider the hydrodynamic excitations in the QCD matter rotating with a constant vorticity $(\boldsymbol{B}=0, \boldsymbol{\Omega} \neq 0)$. Finally in the most general case, the hydrodynamic modes are studied in a rotating QCD fluid coupled to an external magnetic field. Let us mention that in the last part of the paper, we take into account the effect of dissipation in the special case where $\boldsymbol{\Omega}=0$ and $\mu_{5}=0$. Let us note that the effect of dissipation has been also considered in [43] to study the induction of an axial current in the direction of the electric field in thermal QED plasma.

\section{QCD FLUID COUPLED TO EXTERNAL MAGNETIC FIELD}

In this section, we consider a QCD-type fluid coupled to an external magnetic field and compute the spectrum of its hydrodynamic excitations in detail. After deriving the covariant linearized equations, we divide our computations into two parts. First, we consider pure scalar perturbations, and then in another subsection we take the mixed scalarvector perturbations under study. To be more complete and clear, we also discuss the Riemann invariants of the fluid and show which coherent combination of perturbations corresponds to each hydrodynamic excitation.

\section{A. Equations of motion linearized}

Let us consider the hydrodynamic field defined in (9) slightly deviated from its thermodynamic value as the following:

$$
\phi_{a}+\delta \phi_{a}=\left(T+\delta T, \mathbf{0}+\boldsymbol{\pi}, \mu+\delta \mu, \mu_{5}+\delta \mu_{5}\right) .
$$

To first order in $\delta$ variations, the equations of motion may be covariantly written as

$$
M_{a b}^{\boldsymbol{B}}(\boldsymbol{k}, \omega) \delta \phi_{b}(\boldsymbol{k}, \omega)=0
$$

with $M_{a b}^{B}$ given by (see A 2 for thermo coefficients) 


$$
\left[\begin{array}{cccc}
-i \alpha_{1} \omega & i k_{j} & -i \alpha_{2} \omega & -i \alpha_{3} \omega \\
i \alpha_{1} v_{s}^{2} k^{i} & -i \omega \delta_{j}^{i}-i \frac{\xi}{2 \bar{w}}\left(\boldsymbol{B} \cdot \boldsymbol{k} \delta_{j}^{i}-B_{j} k^{i}\right)-\frac{\bar{n}}{\bar{w}} \epsilon_{j l}^{i} B^{l} & i \alpha_{2} v_{s}^{2} k^{i} & i \alpha_{3} v_{s}^{2} k^{i} \\
-i \beta_{1} \omega+\left(\frac{\partial \xi_{B}}{\partial T}\right) i \boldsymbol{B} \cdot \boldsymbol{k} & \frac{\bar{n}}{\bar{w}} i k_{j}-\frac{\xi_{B}}{\bar{w}} i \omega B_{j} & -i \beta_{2} \omega+\left(\frac{\partial \xi_{B}}{\partial \mu}\right) i \boldsymbol{B} \cdot \boldsymbol{k} & -i \beta_{3} \omega+\left(\frac{\partial \xi_{B}}{\partial \mu_{5}}\right) i \boldsymbol{B} \cdot \boldsymbol{k} \\
-i \gamma_{1} \omega+\left(\frac{\partial \xi_{5 B}}{\partial T}\right) i \boldsymbol{B} \cdot \boldsymbol{k} & \frac{\bar{n}_{5}}{\bar{w}} i k_{j}-\frac{\xi_{5 B}}{\bar{w}} i \omega B_{j} & -i \gamma_{2} \omega+\left(\frac{\partial \xi_{5 B}}{\partial \mu}\right) i \boldsymbol{B} \cdot \boldsymbol{k} & -i \gamma_{3} \omega+\left(\frac{\partial \xi_{5 B}}{\partial \mu_{5}}\right) i \boldsymbol{B} \cdot \boldsymbol{k}
\end{array}\right] .
$$

The superscript $\boldsymbol{B}$ on $M_{a b}^{\boldsymbol{B}}$ here refers to this point that in this section we are studying modes in the presence of an external magnetic field. In the next two sections, we change the superscripts to $\boldsymbol{\Omega}$ and $\boldsymbol{B} \boldsymbol{\Omega}$ respectively. As can be obviously seen above, none of the elements of matrix $M_{a b}^{\boldsymbol{B}}$ vanish in general. It means that each of the hydrodynamic excitations in this system might be a coherent excitation of all scalar and vector perturbations. Via computing the Riemann invariants, however, one can exactly determine the type of each propagating mode in the fluid [44].

\section{B. Characteristics and Riemann invariants}

Before starting to compute the hydrodynamic modes, let us briefly review the notion of the characteristics and the Riemann invariants in fluid dynamics. As is well-known, in a fluid whose space of states is $d$-dimensional (in our case $d=6$ ), there exist, in general, $d$ characteristics or equivalently $d$ hydrodynamic waves. These characteristics describe the different ways through which a small perturbation in the state of fluid may propagate in the state-space. For each of the characteristics, there is a corresponding family of integral curves in the state-space. Those perturbations that propagate only through the curves of one characteristic family correspond to the Riemann invariants [33]. Thus, the Riemann invariant $\mathcal{R}_{i}$ associated with the hydrodynamic mode $\omega_{i}$ satisfies the following equation:

$$
\left(\partial_{t}+\boldsymbol{v}_{i} \cdot \boldsymbol{\nabla}\right) \mathcal{R}_{i}=0, \quad i=1, \ldots, 6
$$

where $\boldsymbol{v}_{i}$ is the velocity of $i^{t h}$ mode, namely $\omega_{i}$.

In order to determine the Riemann invariants, one assumes that the linear equations of perturbations may be written as follows:

$$
\partial_{t} \delta \phi_{a}(\boldsymbol{k}, t)+D_{a b}^{\boldsymbol{B}}(\boldsymbol{k}) \delta \phi_{b}(\boldsymbol{k}, t)=0 .
$$

Firstly, it is needed to compute the eigenvalues of the matrix $D^{B}$ as the characteristics or, equivalently, the hydrodynamic modes. To this end one has to find the roots of the determinants of the matrix $M^{B}$, perturbatively order by order, in derivative expansion. The structure of eigenmodes is as follows:

$$
\omega_{i}^{\boldsymbol{B}}(\boldsymbol{k}, \boldsymbol{B})=\omega_{i}^{\boldsymbol{B}(1)}(\boldsymbol{k}, \boldsymbol{B})+\omega_{i}^{\boldsymbol{B}(2)}(\boldsymbol{k}, \boldsymbol{B})+\cdots .
$$

In the above equation, $\omega_{i}^{\boldsymbol{B}(1)}$ and $\omega_{i}^{\boldsymbol{B}(2)}$, are the zero and first order derivative parts of dispersion relation. More explicitly, if we get $\epsilon_{f}$ as the parameter which counts the number of derivatives, we would have

$$
\begin{aligned}
\omega_{i}^{B(1)}\left(\epsilon_{f} \boldsymbol{k}, \epsilon_{f} \boldsymbol{B}\right) & =\epsilon_{f} \omega_{i}^{\boldsymbol{B}(1)}(\boldsymbol{k}, \boldsymbol{B}) \\
\omega_{i}^{\boldsymbol{B}(2)}\left(\epsilon_{f} \boldsymbol{k}, \epsilon_{f} \boldsymbol{B}\right) & =\epsilon_{f}^{2} \omega_{i}^{\boldsymbol{B}(2)}(\boldsymbol{k}, \boldsymbol{B}) \\
\cdots & =\cdots .
\end{aligned}
$$

The next step is to compute the eigenvectors of matrix $D^{B}$. Then the Riemann invariant associated with each of these vectors is the special scalar combination of the components of $\delta \phi_{a}$ which remains invariant along the integral curve generated by that eigenvector in the spacestate. It should be denoted that, in Fourier space, $D_{a b}^{\boldsymbol{B}}=$ $M_{a b}^{\boldsymbol{B}}+i \omega \delta_{a b}$ where $M_{a b}^{\boldsymbol{B}}$ was defined in (14).

\section{Eigenvectors}

In general, a hydrodynamic mode with dispersion relation $\omega_{i}=\omega_{i}(k)$ is characterized as a plane wave

$$
\delta \phi_{a i}(t, \boldsymbol{x})=\delta \tilde{\phi}_{a i} e^{-i \omega_{i}(k) t+i \boldsymbol{k} . x}
$$

where the amplitude of the wave, namely $\delta \tilde{\phi}_{i}$, is referred to as the $i^{t h}$ eigenvector of the matrix $M^{\boldsymbol{B}}$. The basis for our six-dimensional state-space is made out of $\delta T, \delta \pi, \delta \mu$, and $\delta \mu_{5}$. Thus, a general eigenvector of the matrix $M^{\boldsymbol{B}}$ takes the following form in this basis:

$\delta \tilde{\phi}_{a i}=\left(\delta \tilde{\phi}_{1}, \delta \tilde{\phi}_{2,3,4}, \delta \tilde{\phi}_{5}, \delta \tilde{\phi}_{6}\right)_{i}:=\left(\delta \tilde{\phi}_{T}, \delta \tilde{\phi}_{\pi}, \delta \tilde{\phi}_{\mu}, \delta \tilde{\phi}_{\mu_{5}}\right)_{i}$.

\section{Different sectors of the propagation}

Depending on the type of perturbations carrying by a mode, one can characterize the eigenvectors into the scalar, vector, or mixed subsectors. As before, we use scalar and vector terminologically for the representations of the $S O(3)$ 


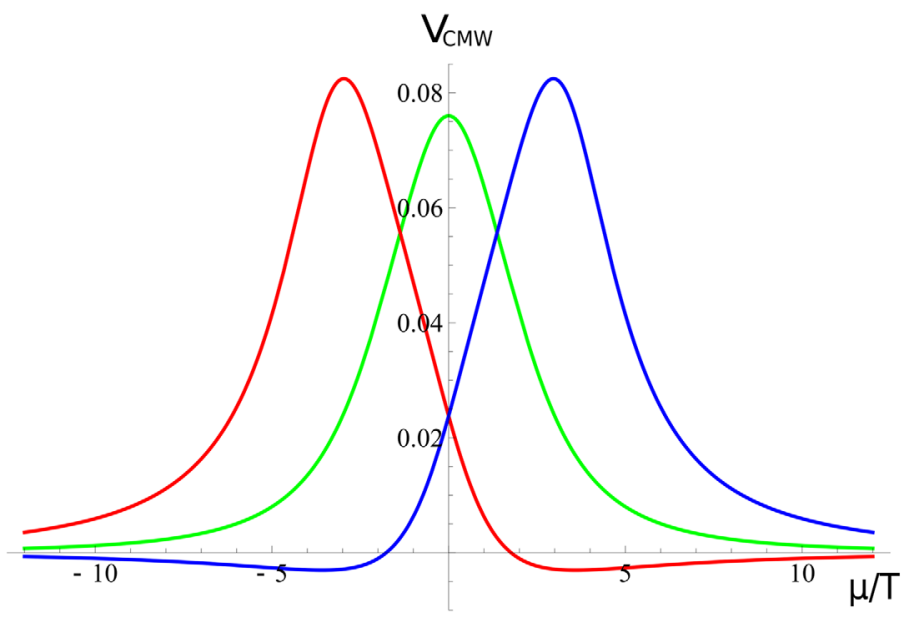

(a) $\frac{\mu_{5}}{T}=3$ and $\frac{B}{T^{2}}=0.5$

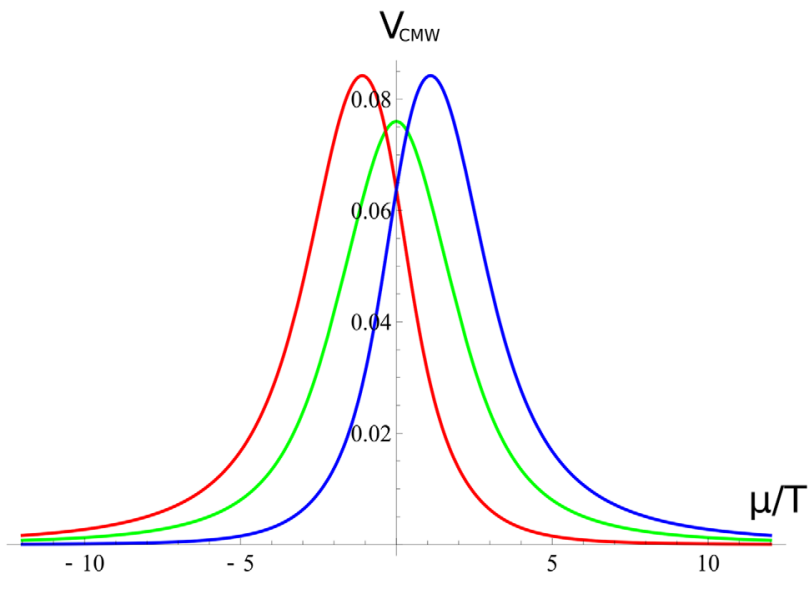

(b) $\frac{\mu_{5}}{T}=1$ and $\frac{B}{T^{2}}=0.5$

FIG. 1. The velocities of the nondegenerate CMHWs (red $=v_{C M H W 1}$ and blue $=-v_{C M H W 2}$ ) compared to the case $\mu_{5}=0$ (green). In both panels, we have taken $\boldsymbol{k} \| \boldsymbol{B}$.

group orthogonal to the local velocity of the fluid at each point. Therefore, the scalar modes are those that carry the perturbations of the $\delta T, \delta \mu$, or $\delta \mu_{5}$, while a vector mode carries a combination of the momentum perturbations $\pi$ 's. It is clear that a mixed scalar-vector mode carries scalar perturbations together with the vector perturbations.

Our computations show that in a general fluid with two axial and vector currents, no pure vector type hydrodynamic mode propagates. We find that the six hydrodynamic modes of the fluid, obtained from the matrix $M^{B}$ in Sec. III A, are divided into the following two sets:

(i) two scalar modes (22).

It turns out that these two modes vanish at zeroth order, namely in ideal hydrodynamics, and appear from the first order in derivative expansion,

$$
\omega_{1,2}^{\boldsymbol{B}}=0+\omega_{1,2}^{\boldsymbol{B}(2)}+O\left(\partial^{3}\right) .
$$

(ii) four mixed scalar-vector modes (35) and (36).

These four modes are, in general, nonvanishing in both zero and first orders of derivatives,

$$
\omega_{3,4,5,6}^{\boldsymbol{B}}=\omega_{3,4,5,6}^{\boldsymbol{B}(1)}+\omega_{3,4,5,6}^{\boldsymbol{B}(2)}+O\left(\partial^{3}\right) .
$$

In the following two subsections we give the dispersion relations and also discuss the nature of the above 1 and 2 sets separately.

\section{Scalar sector: Chiral-magnetic-heat waves}

Among the six eigenmodes, two modes vanish at ideal (zero) order. The first nonvanishing contribution to their dispersion relation comes from the first-order corrections of constitutive relations. One finds

$$
\omega_{1,2}^{\boldsymbol{B}}(k)=-\frac{\mathcal{A}_{1} \pm \sqrt{\mathcal{A}_{1}^{2}-\mathcal{A}_{2} \mathcal{E}}}{\mathcal{E}} \boldsymbol{B} \cdot \boldsymbol{k}
$$

where the $\omega_{1}^{\boldsymbol{B}}$ and $\omega_{2}^{\boldsymbol{B}}$ refer to + and - in front of the square root, respectively. We call the velocity of these modes $v_{C M H W 1}$ and $v_{C M H W 2}$. In the above formula, we have defined

$$
\mathcal{E}=-\epsilon^{i j k} \alpha_{i} \beta_{j} \gamma_{k} \quad\left(\epsilon^{123}=1\right)
$$

with anomaly expressions [45]

$$
\begin{gathered}
\mathcal{A}_{0}=\frac{n \mu}{w} \alpha_{[1} \gamma_{2]}+\frac{n_{5} \mu_{5}}{w} \alpha_{[1} \beta_{3]}-\frac{n \mu_{5}}{w} \alpha_{[1} \gamma_{3]}-\frac{n_{5} \mu}{w} \alpha_{[1} \beta_{2]}, \\
\mathcal{A}_{1}=\frac{\mathcal{C}}{2}\left(\alpha_{[3} \beta_{1]}+\alpha_{[2} \gamma_{1]}+\frac{2 \mu \mu_{5}}{w} \mathcal{E}+\mathcal{A}_{0}\right), \\
\mathcal{A}_{2}=\mathcal{C}^{2} \alpha_{1}\left(1-\frac{n \mu+n_{5} \mu_{5}}{w}\right) \\
+\mathcal{C}^{2} \frac{\mu \mu_{5}}{w}\left(\alpha_{[3} \beta_{1]}+\alpha_{[2} \gamma_{1]}+\frac{\mu \mu_{5}}{w} \mathcal{E}+\mathcal{A}_{0}\right) .
\end{gathered}
$$

It is worth mentioning that, depending on the values of $\mu$ and $\mu_{5}$, the overall sign of each mode in (22) might be either positive or negative. The probable minus sign in the dispersion relation means that in order to have positive frequency, the wave has to propagate in the opposite direction of a mode with positive sign. This relative behavior can be clearly seen in Fig. 1. The same argument goes on for other similar situations in this paper.

The eigenvectors associated with modes (22) are

$$
\delta \tilde{\phi}_{1,2}^{B}=\left(r \frac{\alpha_{2}}{\alpha_{1}}+s \frac{\alpha_{3}}{\alpha_{1}}, \mathbf{0},-r,-s\right),
$$

with $r$ and $s$ being arbitrary parameters. Let us mention that these vectors have been given up to zero order in derivative 
expansion. The ambiguity in fully specifying these eigenvectors is related to the point that to this order, the modes $\omega_{1,2}^{\boldsymbol{B}}$ are degenerate, both with the eigenvalue being zero. Thus, we have the freedom to choose any two arbitrary vectors with the above form as the corresponding eigenvectors. One can find two linearly independent orthogonal eigenvectors $\delta \tilde{\phi}_{1,2}^{B}$ as follows. First we take two vectors from the subspace spanned by (27) by choosing $r=1, s=0$ and $r=0, s=1$,

$$
\begin{aligned}
& n_{1}^{\boldsymbol{B}}=\left(\frac{\alpha_{2}}{\alpha_{1}}, \mathbf{0},-1,0\right) \\
& n_{2}^{\boldsymbol{B}}=\left(\frac{\alpha_{3}}{\alpha_{1}}, \mathbf{0},-1,0\right) .
\end{aligned}
$$

Now we project $n_{2}^{\boldsymbol{B}}$ on the direction of $n_{1}^{\boldsymbol{B}}$ and then subtract the projection vector from $n_{2}^{\boldsymbol{B}}$. The resultant vector, $n_{3}^{\boldsymbol{B}}$, is perpendicular to $n_{1}^{B}$. Therefore, we can get this vector together with $n_{1}^{B}$ as the two eigenvectors corresponding to CMWVs,

$$
\begin{gathered}
\delta \tilde{\phi}_{1}^{\boldsymbol{B}}=n_{1}^{\boldsymbol{B}}=\left(\frac{\alpha_{2}}{\alpha_{1}}, \mathbf{0},-1,0\right) \\
\delta \tilde{\phi}_{2}^{\boldsymbol{B}}=n_{3}^{\boldsymbol{B}}=\left(\frac{\frac{\alpha_{3}}{\alpha_{1}}}{1+\left(\frac{\alpha_{2}}{\alpha_{1}}\right)^{2}}, \mathbf{0}, \frac{\frac{\alpha_{3} \alpha_{2}}{\alpha_{1}^{2}}}{1+\left(\frac{\alpha_{2}}{\alpha_{1}}\right)^{2}},-1\right) .
\end{gathered}
$$

That the amplitude of these waves is spanned by $\delta T, \delta n$, and $\delta n_{5}$ in the 3-dimensional scalar subspace of state-space means that these modes are scalar-type. Let us recall that by using the standard thermodynamic transformations (see Appendix A 1) one can alternatively express the eigenvectors (27) in terms of another set of fluctuations, e.g., $\delta \epsilon, \delta n$, and $\delta n_{5}$. So the modes $\omega_{1,2}^{\boldsymbol{B}}$ are in fact the coherent perturbations of energy, vector, and axial charge currents.

Let us remind that while the well-known CMWs [23] carry exclusively the perturbations of axial and vector currents, the waves found here carry the energy (temperature) perturbations as well. For this reason, we refer to them as the chiralmagnetic-heat wave (CMHW). In order to make clear the feature of CMHWs, let us go back and consider the eigenvectors (27). The thermodynamic coefficients $\alpha_{2}=$ $\partial \epsilon / \partial \mu$ and $\alpha_{3}=\partial \epsilon / \partial \mu_{5}$ are nonvanishing at finite vector and axial charge densities, so one would expect only at $n=$ $n_{5}=0$ [23], the temperature perturbation is not carried by the CMHWs [see equation (27)]. In the latter limit, the CMHW changes to CMW. Additionally, while both the leftand right-moving CMWs in [23] are identified with one velocity, the velocity of two CMHWs is not the same; one of them in general moves faster than the other. It is in fact the manifestation of the energy transport by the CMHWs.

Using the exact form of the equation of state, the above discussion can be understood more quantitatively. In a conformal fluid of noninteracting fermions with both vector and axial charges, we have

$$
\begin{aligned}
\epsilon=3 p= & \frac{7 \pi^{2}}{60} T^{4}+\frac{1}{2}\left(\mu^{2}+\mu_{5}^{2}\right) T^{2} \\
& +\frac{1}{4 \pi^{2}}\left(\mu^{4}+6 \mu^{2} \mu_{5}^{2}+\mu_{5}^{4}\right) .
\end{aligned}
$$

In the following, we study CMHWs found above in a fluid with the above equation of state. In Figure 1, we have plotted the dependence of the velocities of the fast and slow CMHWs on $\frac{\mu}{T}$, in two cases, each of which corresponded to a special value of the $\frac{\mu_{5}}{T}$ in equilibrium [46]. In each case, we have also depicted the changes of CMHW at $\mu_{5}=0$ with a green curve. Note that according to (22), CMHWs are not dispersive and this means that the curves presented in this figure are unique for CMHWs with each arbitrary wavelength in the hydrodynamic regime.

As is observed in Fig. 1, for large values of $\frac{\mu_{5}}{T}$, the fast and slow waves may propagate either in opposite or in the same directions (panel a); it depends on the value of $\frac{\mu}{T}$ in a fixed $\frac{B}{T^{2}}$. In the smaller values of $\frac{\mu_{5}}{T}$, however, two CMHWs always propagate opposite to each other (panel b). It should be noted that in both panels, the difference between velocities of the fast and slow CMHWs, is due to presence of a finite axial charge density in the fluid; actually at $\mu_{5}=0$, the coefficient $\mathcal{A}_{1}$ in (22) vanishes and the velocities of both waves become the same.

Another point with Fig. 1 is that each of the fast and slow waves reaches to its maximum velocity when $\mu=\mu_{5}$ or $\mu=-\mu_{5}$ [47]. Consequently, when $\mu_{5}=0$, the velocities of two waves become degenerate with a maximum at $\mu=$ $\mu_{5}=0$ (green curve).

As the last point regarding Fig. 1, we compare two special limits with each other. First suppose $n_{5}=0$ while $n \neq 0$; in this case, as can be clearly seen by green curves in figure, a degenerate CMHW does exist. In the opposite limit when $n_{5} \neq 0$ and $n=0$, again two degenerate CMHWs propagate corresponding to the common $v$ intercept of the blue and red curves in the figure.

Before ending this subsection, let us emphasize that the novelty of our results is not limited to the $\mu_{5} \neq 0$ case. Even at $\mu_{5}=0$, our results are novel since we have considered the fluctuations of energy-momentum as well as the charge fluctuations. To make this point clear, let us consider equation (22). At $\mu_{5}=0$ this equation simplifies to

$$
\omega_{1,2}^{\boldsymbol{B}}= \pm \frac{B k}{2 \pi^{2} \chi} \frac{1-\frac{\mu n}{w}}{\sqrt{1-\frac{\mu n}{w}-\frac{n}{\chi w}\left(\frac{n}{c_{s}^{2}}-\chi \mu\right)}}
$$

with $\chi=\partial n / \partial \mu$. This result differs clearly from the CMW

$$
\omega= \pm \frac{B k}{2 \pi^{2} \chi}
$$


obtained in [13] by turned off energy-momentum fluctuations [48].

\section{Mixed scalar-vector sector}

In addition to two scalar modes given in previous subsection, matrix $M_{a b}^{\boldsymbol{B}}$ has another four perturbative roots corresponding to four different hydrodynamic modes. In contrast to scalar sector modes, the four new modes are present even in ideal (zero-order) hydrodynamics. Up to first order in derivative correction of constitutive relations, or equivalently up to second order in derivative expansion of dispersion relations, we obtain $\omega_{3,4}^{\boldsymbol{B}}=$ $\omega_{3,4}^{B(1)}+\omega_{3,4}^{B(2)}$ with

$$
\begin{aligned}
& \omega_{3,4}^{\boldsymbol{B}(1)}= \pm \frac{1}{\sqrt{2}} \sqrt{c_{s}^{2} k^{2}+\Omega_{L}^{2}+\sqrt{\left(c_{s}^{2} k^{2}+\Omega_{L}^{2}\right)^{2}-4 c_{s}^{2} k^{2} \Omega_{L}^{2} \cos ^{2} \theta}} \\
& \omega_{3,4}^{\boldsymbol{B}(2)}=-\frac{\Omega_{L}^{2}\left(\left(\omega_{3,4}^{\boldsymbol{B}(1)}\right)^{2}-c_{s}^{2} k^{2} \cos ^{2} \theta\right) \cos \theta}{\left(c_{s}^{2} k^{2}+\Omega_{L}^{2}\right)\left(\omega_{3,4}^{\boldsymbol{B}(1)}\right)^{2}-2 c_{s}^{2} k^{2} \Omega_{L}^{2} \cos ^{2} \theta}\left(\frac{\xi B}{2 w}\right) k
\end{aligned}
$$

and $\omega_{5,6}^{\boldsymbol{B}}=\omega_{5,6}^{\boldsymbol{B}(1)}+\omega_{5,6}^{\boldsymbol{B}(2)}$ with

$$
\begin{aligned}
& \omega_{5,6}^{\boldsymbol{B}(1)}= \pm \frac{1}{\sqrt{2}} \sqrt{c_{s}^{2} k^{2}+\Omega_{L}^{2}-\sqrt{\left(c_{s}^{2} k^{2}+\Omega_{L}^{2}\right)^{2}-4 c_{s}^{2} k^{2} \Omega_{L}^{2} \cos ^{2} \theta}} \\
& \omega_{5,6}^{\boldsymbol{B}(2)}=-\frac{\Omega_{L}^{2}\left(\left(\omega_{5,6}^{\boldsymbol{B}(1)}\right)^{2}-c_{s}^{2} k^{2} \cos ^{2} \theta\right) \cos \theta}{\left(c_{s}^{2} k^{2}+\Omega_{L}^{2}\right)\left(\omega_{5,6}^{\boldsymbol{B}(1)}\right)^{2}-2 c_{s}^{2} k^{2} \Omega_{L}^{2} \cos ^{2} \theta}\left(\frac{\xi B}{2 w}\right) k
\end{aligned}
$$

where $\cos \theta=\hat{\boldsymbol{B}} \cdot \hat{\boldsymbol{k}}$. In the equations given above, $\Omega_{L}$ is the Larmor frequency as being

$$
\Omega_{L}=\frac{n B}{w} .
$$

Considering (17), the outer square root in $\omega_{3,4,5,6}^{(1)}$ turns out to be of order $O(\partial)$. In the case of $\omega_{3,4,5,6}^{(2)}$ however, more clarification is needed to understand why it is of order $O\left(\partial^{2}\right)$. Note that under rescaling $k \rightarrow \epsilon_{f} k$ and $B \rightarrow \epsilon_{f} B$, the fraction part in these relations behaves as a zero-order object (fraction $\rightarrow \epsilon_{f}^{0}$ fraction). Thus, the same as for $\frac{\xi B}{w} k$, the derivative order of $\omega_{3,4,5,6}^{(2)}$ is $O\left(\partial^{2}\right)$.

The eigenvectors corresponding to the above four modes are as the following:

$$
\begin{aligned}
\delta \tilde{\phi}_{i}^{\boldsymbol{B}} & =\left(\delta T, \delta \boldsymbol{\pi}, \delta \mu, \delta \mu_{5}\right) \\
& =\left(1,-\frac{w \mathcal{E} \omega_{i}^{(1)} \boldsymbol{k}}{C_{2} \boldsymbol{k}^{2}}+\frac{n \mathcal{E}\left(i\left(\omega_{i}^{\boldsymbol{B}(1)}\right)^{2}(\boldsymbol{B} \times \boldsymbol{k})+\frac{n}{w} \omega_{i}^{\boldsymbol{B}(1)}(\boldsymbol{B} \cdot \boldsymbol{k}) \boldsymbol{B}-\frac{n}{w} \omega_{i}^{\boldsymbol{B}(1)}(\boldsymbol{B} \cdot \hat{\boldsymbol{k}})^{2} \boldsymbol{k}\right)}{C_{2}\left(\left(\omega_{i}^{\boldsymbol{B}(1)}\right)^{2} \boldsymbol{k}^{2}-n^{2} / w^{2}(\boldsymbol{B} \cdot \boldsymbol{k})^{2}\right)}, \frac{C_{1}}{C_{2}}, \frac{C_{3}}{C_{2}}\right)
\end{aligned}
$$

with

$$
\begin{aligned}
& C_{1}=n \alpha_{[1} \gamma_{3]}-n_{5} \alpha_{[1} \beta_{3]}-w \beta_{[1} \gamma_{3]} \\
& C_{2}=n \alpha_{[3} \gamma_{2]}-n_{5} \alpha_{[3} \beta_{2]}-w \beta_{[3} \gamma_{2]} \\
& C_{3}=n \alpha_{[2} \gamma_{1]}-n_{5} \alpha_{[2} \beta_{1]}-w \beta_{[2} \gamma_{1]} .
\end{aligned}
$$

Let us denote that the above eigenvectors have generally nonvanishing scalar and vector components in the statespace. For this reason, we refer to the current sector as the scalar-vector sector.

At zero order in derivative expansion, each of these modes is a mixture of the ordinary sound with Larmor frequency, reminiscent of the magnetosonic waves in the ideal magnetohydrodynamics [50]. At first order in derivative expansion, these four mixed modes get corrections proportional to the magnetic field. The situation is actually analogous to what appears in the dispersion relation of chiral Alfvén waves (CAWs) in a chiral fluid of single right-handed fermions $[29,30]$. As a result, one may refer to the scalar-vector sector modes as the mixed sound-Alfvén waves. In the following, it becomes more clear why this terminology is used. 
In the special case of propagation in the direction of the magnetic field, $\boldsymbol{B} \| \boldsymbol{k}$, the above scalar-vector modes become distinguishable with the following velocities:

$$
\begin{gathered}
\omega_{3,4}^{\boldsymbol{B}}= \pm c_{s} k \rightarrow v_{3,4}^{\boldsymbol{B}}= \pm c_{s} \\
\omega_{5,6}^{\boldsymbol{B}}= \pm \frac{n B}{w}-\frac{\xi}{2 w} B . k \rightarrow v_{5,6}^{\boldsymbol{B}}=-\frac{\xi}{2 w} B .
\end{gathered}
$$

Clearly the modes 5 and 6 are two gapped chiral waves propagating parallel with the magnetic field. These are the counterpart of CAWs in a chiral fluid with single chirality, recently found in $[29,30]$. The terminology, choosing by reference [29], might seem a little misleading; there are some differences between CAWs and standard Alfvén waves in magnetohydrodynamics (MHD). First, the Alfvén waves in MHD are gapless while in Chiral fluids they are gapped. Second and more important, it is the dynamics of Maxwell fields which lead the Alfvén waves to propagate in MHD, while in our case, a nondynamical magnetic field is able to couple to the local fluctuations of vorticity in the chiral fluid and excites a collective motion parallel to itself, referred to as the chiral Alfvén wave in [29]. Despite knowing these differences, since waves 5 and 6 propagate parallel to the magnetic field, we follow [29] and call them the chiral Alfvén waves.

In another limit, when $\boldsymbol{B} \perp \boldsymbol{k}$, we have only two gapped plasmon modes [51],

$$
\begin{gathered}
\omega_{3,4}^{\boldsymbol{B}}= \pm \sqrt{c_{s}^{2} k^{2}+\Omega_{L}^{2}} \rightarrow v_{3,4}^{\boldsymbol{B}}= \pm \frac{c_{s}}{\sqrt{1+\frac{\Omega_{L}^{2}}{c_{s}^{2} k^{2}}}} \\
\omega_{5,6}^{\boldsymbol{B}}=0 .
\end{gathered}
$$

As one naturally expects, analogous to the case of a chiral fluid with single chirality [30], the anomaly effects cannot be detected in the directions transverse to the magnetic field here. The only modes propagating in transverse directions are the magnetosonic waves. By magnetosonic waves here, however, we do not mean exactly the familiar magnetosonic waves in the ideal magnetohydrodynamics. As is well-known in magnetohydrodynamics, the pressure perturbations produced by Maxwell dynamics intensify the fluid pressure perturbations, resulting in an excess in the velocity of sound. While in the latter case, the pressure perturbations are intensified due to the compression and rarefaction of the magnetic field lines; in our case however, a constant magnetic field exerts opposite external Lorentz forces on momentum perturbations and decreases the hydrodynamical pressure.

In summary, we observe that the modes in the scalar-vector sector are, in general, the coherent perturbation of all six hydrodynamic fields. They are mixed-sound-Alfvén waves.

Before ending this section, let us separate the new results of the paper in this part from their well-known counterparts in the literature. To our knowledge, the hydrodynamic excitations of a chiral fluid with both vector and axial currents was studied only in the absence of momentum perturbations before. In other words, the hydrodynamic excitations had been computed only for a "Forced" QCD fluid before. None of the six modes (22), (35), and (36) were found in previous studies. In the case of CMHWs (22), even at $\mu_{5}=0$, our result, namely (33), was not wellknown before. Only at $\mu=\mu_{5}=0$, in which the temperature perturbations decouple from that of vector and axial currents, does the result, namely (34), exist in the literature. The latter is nothing but the well-known chiral magnetic wave. In the case of mixed vector modes (35) and (36), the novelty of our results is twofold; first, that our results are covariant by this means that we have found the dispersion relation for propagation in every arbitrary direction with respect to the external magnetic field. Second, even in $\boldsymbol{B} \| \boldsymbol{k}$, the gapped CAW (43) found in the current paper was not found before, although in the case of single chirality fluid such mode had been found firstly in [30] and afterward in [52,53]. Furthermore, nowhere in the literature have we seen the eigenvectors (27) and (38) associated with hydrodynamic modes in a QCD type fluid.

\section{ROTATING QCD FLUID}

In this part, we consider a QCD fluid, rotating with constant vorticity $\boldsymbol{\Omega}$, in the absence of electromagnetic fields, with the four-velocity

$$
u^{\mu}=\gamma(1, \boldsymbol{\Omega} \times \boldsymbol{x}) .
$$

In what follows, we consider the regime $\Omega r \ll 1$, where $r$ is the distance from the axis of the rotation. In this regime the Lorentz factor may be expanded as $\gamma=1+O\left((\Omega r)^{2}\right)$, so the vorticity computed in equilibrium up to first order in $O(\Omega r)$ becomes

$$
\omega^{\mu}=(0, \boldsymbol{\Omega}) .
$$

\section{A. Equations of motion linearized}

Let us take the small deviation of hydrodynamic fields (9) away from their equilibrium values as follows:

$$
\delta \phi_{a}=\left(\delta T, \pi, \delta \mu, \delta \mu_{5}\right) .
$$

In order to linearize the equations of motion, we have to expand the equations (1) around the equilibrium state,

$$
\begin{aligned}
u^{\mu} & =(1, \boldsymbol{\Omega} \times \boldsymbol{x}) \quad \Omega r \ll 1, \\
T & =\text { Const }, \quad \mu=\text { Const }, \quad \mu_{5}=\text { Const },
\end{aligned}
$$

and keep the terms up to first order in $\delta \phi_{a}$ fields. Among all terms, there is a delicate point regarding the expansion of the vorticity terms of (4) and (5) around equilibrium which deserves to be explained in detail. Consider the velocity of a fluid perturbed by $\delta u^{\mu}=\left(\delta u^{0}, \delta \boldsymbol{u}\right)$ as 


$$
u^{\mu}+\delta u^{\mu}=\left(1+\delta u^{0}, \boldsymbol{\Omega} \times \boldsymbol{x}+\delta \boldsymbol{u}\right) .
$$

Demanding the above velocity to satisfy the relativistic normalization $u^{\mu} u_{\mu}=-1$, the zero component of the perturbation is immediately fixed

$$
\delta u^{0}=(\boldsymbol{\Omega} \times \boldsymbol{x}) \cdot \delta \boldsymbol{u} .
$$

Thus, to first order in perturbations, the vorticity takes the following form: $\omega^{\mu}+\delta \omega^{\mu}=\left(\frac{\xi}{w}(\delta \boldsymbol{\pi} \cdot \boldsymbol{\Omega}), \boldsymbol{\Omega}+\frac{1}{2 w} \boldsymbol{\nabla} \times \delta \boldsymbol{\pi}\right), \quad \Omega r \ll 1$.

Using the above expression, the linearized equations of motion may be covariantly written as

$$
M_{a b}^{\boldsymbol{\Omega}}(\boldsymbol{k}, \omega) \delta \phi_{b}(\boldsymbol{k}, \omega)=0,
$$

with $M_{a b}^{\boldsymbol{\Omega}}$ given by

$$
\left[\begin{array}{cccc}
-i \alpha_{1} \omega & i k_{j} & -i \alpha_{2} \omega & -i \alpha_{3} \omega \\
i \alpha_{1} v_{s}^{2} k^{i} & -i \omega \delta_{j}^{i}-\epsilon_{j l}^{i} \Omega^{l} & i \alpha_{2} v_{s}^{2} k^{i} & i \alpha_{3} v_{s}^{2} k^{i} \\
-i \beta_{1} \omega+\left(\frac{\partial \xi}{\partial T}\right) i \boldsymbol{\Omega} \cdot \boldsymbol{k} & \frac{\bar{n}}{\bar{w}} i k_{j}-\frac{2 \xi}{\bar{w}} i \omega \boldsymbol{\Omega}_{j} & -i \beta_{2} \omega+\left(\frac{\partial \xi}{\partial \mu}\right) i \boldsymbol{\Omega} \cdot \boldsymbol{k} & -i \beta_{3} \omega+\left(\frac{\partial \xi}{\partial \mu_{5}}\right) i \boldsymbol{\Omega} \cdot \boldsymbol{k} \\
-i \gamma_{1} \omega+\left(\frac{\partial \xi_{5}}{\partial T}\right) i \boldsymbol{\Omega} \cdot \boldsymbol{k} & \frac{\bar{n}_{5}}{\bar{w}} i k_{j}-\frac{2 \xi_{5}}{\bar{w}} i \omega \boldsymbol{\Omega}_{j} & -i \gamma_{2} \omega+\left(\frac{\partial \xi_{5}}{\partial \mu}\right) i \boldsymbol{\Omega} \cdot \boldsymbol{k} & -i \gamma_{3} \omega+\left(\frac{\partial \xi_{5}}{\partial \mu_{5}}\right) i \boldsymbol{\Omega} \cdot \boldsymbol{k}
\end{array}\right] .
$$

At this moment, since all the components of the matrix $M_{a b}^{\boldsymbol{\Omega}}$ are nonvanishing, one may think that each of the characteristics of the fluid is a coherent perturbation of all six scalar and vector hydrodynamic variables. We will show in the following that, in fact, two of the characteristics are scalar type, while the other four are mixed scalar-vector perturbations.

\section{B. Hydrodynamic modes}

Computing the eigenvalues of matrix $M_{a b}^{\mathbf{\Omega}}+\omega \mathbf{1}_{a b}$, or equivalently the roots of $\operatorname{det} M_{a b}^{\mathbf{\Omega}}=0$, we find six independent hydrodynamic modes of the fluid. Our computations show that in the rotating fluid, two sets of hydrodynamic modes are present:

(1) two scalar modes (56).

It turns out that these two modes vanish at zeroth order, namely in ideal hydrodynamics, and just appear from the first order in derivative expansion,

$$
\omega_{1,2}^{\mathbf{\Omega}}=0+\omega_{1,2}^{\mathbf{\Omega}(2)}+O\left(\partial^{3}\right) .
$$

(2) four scalar-vector modes (61) and (62).

In contrast to modes in the scalar sector, these four modes are vanishing at first order, contributing at zero order,

$$
\omega_{3,4,5,6}^{\mathbf{\Omega}}=\omega_{3,4,5,6}^{\boldsymbol{\Omega}(1)}+0+O\left(\partial^{3}\right) .
$$

In the following two subsections we give the dispersion relations and also discuss the nature of the above 1 and 2 sets separately.

\section{Scalar sector: Chiral-vortical-heat Waves}

Among the six eigenmodes, two modes vanish at ideal (zero) order. The first nonvanishing contribution to their dispersion relation comes from the first-order corrections of the constitutive relations. One finds

$$
\omega_{1,2}^{\boldsymbol{\Omega}}(k)=-\frac{\mathcal{A}_{3} \pm \sqrt{\mathcal{A}_{3}^{2}-\mathcal{E} \mathcal{A}_{4}}}{\mathcal{E}} \boldsymbol{\Omega} \cdot \boldsymbol{k}+O\left(k^{3}\right)
$$

where the $\omega_{1}^{\Omega}$ and $\omega_{2}^{\Omega}$ refer to + and - in front of the square root, respectively. We also call the velocity of these modes $v_{C V H W 1}$ and $v_{C V H W 2}$, respectively. The coefficients $\mathcal{A}_{3}$ and $\mathcal{A}_{4}$ may be written as polynomials of anomaly coefficients as follows:

$$
\begin{aligned}
& \mathcal{A}_{3}=\mathcal{D} x_{1}+\mathcal{C} x_{2} \\
& \mathcal{A}_{4}=\mathcal{C}^{2} y_{1}+\mathcal{D}^{2} y_{2}+\mathcal{C D} y_{3}
\end{aligned}
$$

with $x_{i} \mathrm{~s}$ given in Appendix A 4 and $y_{i} \mathrm{~s}$ given in Appendix A 5.

The corresponding eigenvectors are

$$
\delta \tilde{\phi}_{1,2}^{\mathbf{\Omega}}=\left(r \frac{\alpha_{2}}{\alpha_{1}}+s \frac{\alpha_{3}}{\alpha_{1}}, \mathbf{0},-r,-s\right), \quad i=1,2
$$

with $r$ and $s$ the arbitrary parameters. Note that we have the freedom to choose any two arbitrary vectors with the above form as the eigenvectors. Analogous to (30) and (31), we can find two linearly independent orthogonal eigenvectors as follows: 


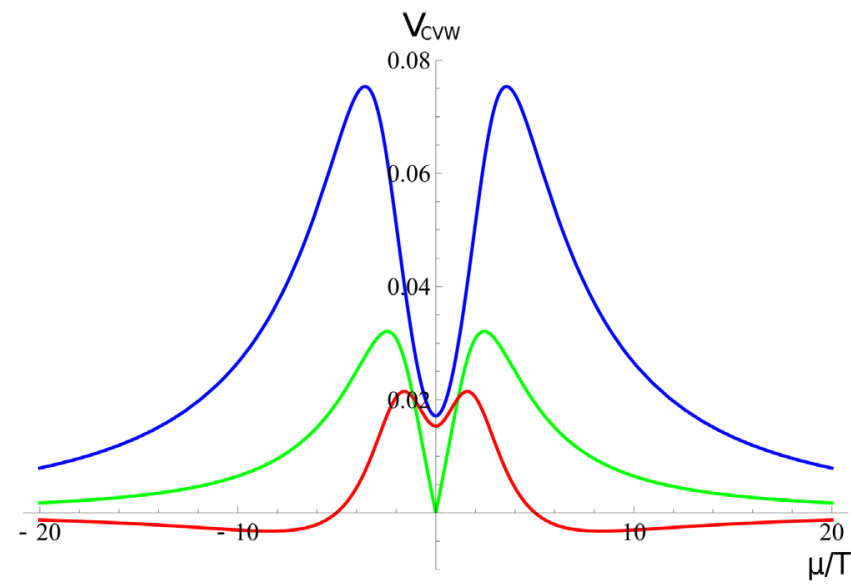

FIG. 2. The velocities of the nondegenerate CVHWs (red $=v_{C V H W 1}$ and blue $\left.=-v_{C V H W 2}\right)$ for $\frac{\mu_{5}}{T}=2$ and $\frac{\Omega}{T}=0.4$ together with the case $\mu_{5}=0$ (green). We have taken $\boldsymbol{k} \| \boldsymbol{\Omega}$.

$$
\begin{gathered}
\delta \tilde{\phi}_{1}^{\boldsymbol{\Omega}}=\left(\frac{\alpha_{2}}{\alpha_{1}}, \mathbf{0},-1,0\right) \\
\delta \tilde{\phi}_{2}^{\boldsymbol{\Omega}}=\left(\frac{\frac{\alpha_{3}}{\alpha_{1}}}{1+\left(\frac{\alpha_{2}}{\alpha_{1}}\right)^{2}}, \mathbf{0}, \frac{\frac{\alpha_{3} \alpha_{2}}{\alpha_{1}^{2}}}{1+\left(\frac{\alpha_{2}}{\alpha_{1}}\right)^{2}},-1\right) .
\end{gathered}
$$

Since these two modes carry the perturbations of temperature together with the vector and axial chemical potentials, we call them chiral-vortical-heat waves [54]. Generally, one of the CVHWs moves faster than the other. Only in the special limit where the fluid is nonchiral, namely when $\mu_{5}=0$ and consequently $\mathcal{A}_{3}=0$, do the velocities of two CVHWs become the same, while definitely propagating in opposite directions [27].

Using the equation of state given in (32), in Fig. 2 we have plotted the dependence of the velocities of the fast and slow CVHWs on $\frac{\mu}{T}$ for a special $\frac{\mu_{5}}{T}$ and $\frac{\Omega}{T}$ in equilibrium. We have also depicted the changes of $\mathrm{CVHW}$ at $\mu_{5}=0$ with a green curve. This plot clearly shows that fast and slow CVHWs do not necessarily propagate in the same direction. As mentioned above, when $\mu_{5}=0$, the velocity of these two waves become equal to each other, independent of the value of $\frac{\mu}{T}$.

It is worth mentioning that the nature of CVHWs is different from that of CMHWs in Fig. 1. Interestingly, while CMHWs can propagate in fluid even at $\mu=\mu_{5}=0$, CVHWs cannot do so. In addition, the velocities of two CMHWs become degenerate when either $\mu=0$ or $\mu_{5}=0$. In contrary, CVHWs have the same velocities only when $\mu_{5}=0$. These observations simply reject the claim that the results in a rotating fluid are similar to those in a fluid coupled to a magnetic field. This difference is not limited to the scalar sector. In the next subsection, we will see that the scalar-vector modes in a rotating chiral fluid have remarkable differences compared to those of a nonrotating chiral fluid in a magnetic field.

\section{Scalar-vector sector}

In addition to the two scalar modes computed in the previous subsection, there are another four modes as follows:

$$
\begin{aligned}
& \omega_{3,4}^{\boldsymbol{\Omega}}= \pm \frac{1}{\sqrt{2}} \sqrt{c_{s}^{2} k^{2}+\Omega^{2}+\sqrt{\left(c_{s}^{2} k^{2}+\Omega^{2}\right)^{2}-4 c_{s}^{2} k^{2} \Omega^{2} \cos ^{2} \theta}}+O\left(k^{3}\right) \\
& \omega_{5,6}^{\boldsymbol{\Omega}}= \pm \frac{1}{\sqrt{2}} \sqrt{c_{s}^{2} k^{2}+\Omega^{2}-\sqrt{\left(c_{s}^{2} k^{2}+\Omega^{2}\right)^{2}-4 c_{s}^{2} k^{2} \Omega^{2} \cos ^{2} \theta}}+O\left(k^{3}\right)
\end{aligned}
$$

with $\cos \theta=\hat{\mathbf{\Omega}} \cdot \hat{\boldsymbol{k}}$.

Considering (17), the square root in all these four modes turns out to be of order $O(\partial)$ and no second-order correction contributes to the dispersion of these modes. Computing the eigenvectors of the matrix $M_{a b}^{\Omega}$, we find for $i=3$, 4, 5, 6:

$$
\begin{aligned}
\delta \tilde{\phi}_{i}^{\boldsymbol{\Omega}} & =\left(\delta T, \delta \boldsymbol{\pi}, \delta \mu, \delta \mu_{5}\right) \\
& =\left(1,-\frac{w \mathcal{E} \omega_{i}^{\boldsymbol{\Omega}} \boldsymbol{k}}{C_{2} \boldsymbol{k}^{2}}+\frac{w \mathcal{E}\left(i\left(\omega_{i}^{\boldsymbol{\Omega}}\right)^{2}(\boldsymbol{\Omega} \times \boldsymbol{k})+\omega_{i}^{\boldsymbol{\Omega}}(\boldsymbol{\Omega} \cdot \boldsymbol{k}) \boldsymbol{\Omega}-\omega_{i}^{\boldsymbol{\Omega}}(\boldsymbol{\Omega} \cdot \hat{\boldsymbol{k}})^{2} \boldsymbol{k}\right)}{C_{2}\left(\left(\omega_{i}^{\mathbf{\Omega}}\right)^{2} \boldsymbol{k}^{2}-(\boldsymbol{\Omega} \cdot \boldsymbol{k})^{2}\right)}, \frac{C_{1}}{C_{2}}, \frac{C_{3}}{C_{2}}\right)
\end{aligned}
$$

with $C_{1}, C_{2}$, and $C_{3}$ given in (39), (40), and (41).

In the special case of propagation in the direction of vorticity, $\boldsymbol{\Omega} \| \boldsymbol{k}$, the above scalar-vector modes become distinguishable from each other as follows:

$$
\omega_{3,4}^{\Omega}= \pm c_{s} k
$$

$$
\omega_{5,6}^{\mathbf{\Omega}}= \pm \Omega \text {. }
$$

Clearly modes 5 and 6 are two standing vortex modes. In another limit when $\boldsymbol{\Omega} \perp \boldsymbol{k}$, we just obtain two sound waves gapped out by the background vorticity, 


$$
\begin{gathered}
\omega_{3,4}^{\boldsymbol{\Omega}}= \pm \sqrt{c_{s}^{2} k^{2}+\Omega_{L}^{2}} \rightarrow v_{3,4}^{\boldsymbol{\Omega}}= \pm \frac{c_{s}}{\sqrt{1+\frac{\Omega_{L}^{2}}{c_{s}^{2} k^{2}}}} \\
\omega_{5,6}^{\boldsymbol{\Omega}}=0 .
\end{gathered}
$$

The only modes propagating in transverse directions are $\omega_{3,4}$, the Coriolis-sound waves, analogous to the magnetosonic waves (44) in the presence of a transverse magnetic field. Note that the anomaly effects cannot be detected in directions transverse to the vorticity.

In summary, when the wave vector is neither parallel nor transverse to the vorticity, the four scalar-vector modes become mixed sound-Coriolis modes, which also disperse when they propagate.

Before ending this section let us separate the new results of the paper in this part from their well-known counterparts in the literature. To our knowledge CVHWs (56) and their corresponding eigenvectors (58) and (63) were not found in previous studies.

\section{ROTATING QCD FLUID COUPLED TO MAGNETIC FIELD}

In this section we consider the general case in which the QCD fluid is both rotating and coupled to an external magnetic field. The associated results are lengthy and complicated, so we just limit ourselves to writing the hydrodynamic eigenmodes formally with a number of coefficients given in the related Appendix.

\section{A. Equations of motion linearized}

The thermodynamic equilibrium state of the fluid may be given by

$$
\begin{aligned}
u^{\mu} & =(1, \Omega \times \boldsymbol{x}) \quad \Omega r \ll 1, \\
T & =\text { Const }, \quad \mu=\text { Const }, \quad \mu_{5}=\text { Const } . \\
\boldsymbol{B} & =\text { Const. }
\end{aligned}
$$

If we slightly perturb the above state as

$$
\phi_{a}+\delta \phi_{a}=\left(T+\delta T, \mathbf{0}+\boldsymbol{\pi}, \mu+\delta \mu, \mu_{5}+\delta \mu_{5}\right)
$$

the linearized equations of motion take the following form

$$
M_{a b}^{B \Omega}(\boldsymbol{k}, \omega) \delta \phi_{a}(\boldsymbol{k}, \omega)=0,
$$

with $M_{a b}^{B \Omega}$ given by (A8) in Appendix A 3 .

As in the previous two sections, two scalar modes together with four mixed scalar-vector modes constituted the full spectrum of the hydrodynamic excitations. As we will see, in the current subsection, the scalar sector include the mixed CMWHWs, while in the scalar-vector sector there exist mixed sound-Alfvén-Coriolis waves.

\section{B. Hydrodynamic modes}

The dispersion relations of the two scalar modes, namely the CMVHWs, in this case are as follows:

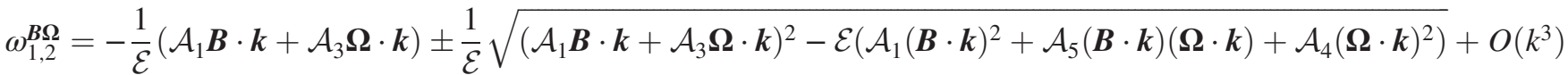

where the new anomaly expression is

$$
\mathcal{A}_{5}=\mathcal{C}^{2} z_{1}+\mathcal{C D T} z_{2}
$$

with $z_{1}$ and $z_{2}$ given in Appendix A 6. These are in fact two waves with different velocities. In the nonchiral limit where
$\mathcal{A}_{1}$ and $\mathcal{A}_{3}$ vanish, the velocities of the two modes become the same.

In the case of the scalar-vector modes, the dispersion relations are very complicated. We first give the dispersion relation of each mode at zero order of hydrodynamic constitutive currents,

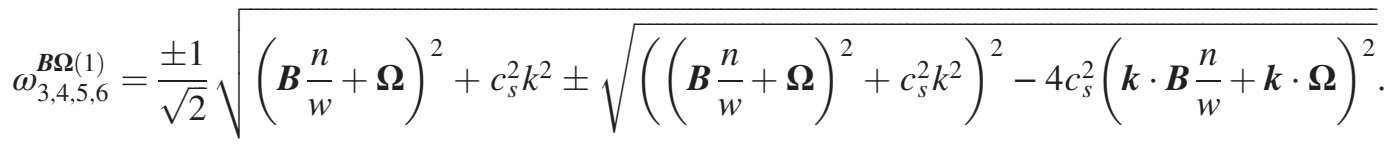

By use of the above four zero-order expressions, one may write the dispersion relations up to first order for $i=3,4,5,6$ as $\omega_{i}^{\boldsymbol{B} \Omega}=\omega_{i}^{\boldsymbol{B} \Omega(1)}+\omega_{i}^{\boldsymbol{B} \boldsymbol{\Omega}(2)}$ with

$$
\omega_{i}^{\boldsymbol{B} \boldsymbol{\Omega}(2)}=-\frac{1}{\mathcal{E}} \frac{\left(\sum_{j} a_{j} \mathbf{s}_{j}\right)\left(\omega_{i}^{\boldsymbol{B} \boldsymbol{\Omega}(1)}\right)^{4}+\left(i b \mathbf{s}_{7}\right)\left(\omega_{i}^{\boldsymbol{B} \boldsymbol{\Omega}(1)}\right)^{3}+\left(\sum_{j, k} c_{j, k} \mathbf{s}_{j} \mathbf{s}_{k}\right)\left(\omega_{i}^{\boldsymbol{B} \boldsymbol{\Omega}(1)}\right)^{2}+\sum_{j, k, l} d_{j, k, l} \mathbf{s}_{j} \mathbf{s}_{k} \mathbf{s}_{l}}{3\left(\omega_{i}^{\boldsymbol{B} \boldsymbol{\Omega}(1)}\right)^{4}-2\left(k^{2} c_{s}^{2}+\left(\boldsymbol{\Omega}+\frac{n}{w} \boldsymbol{B}\right)^{2}\right)\left(\omega_{i}^{\boldsymbol{B} \boldsymbol{\Omega}(1)}\right)^{2}+c_{s}^{2}\left(\boldsymbol{\Omega} \cdot \boldsymbol{k}+\frac{n}{w} \boldsymbol{B} \cdot \boldsymbol{k}\right)^{2}} .
$$




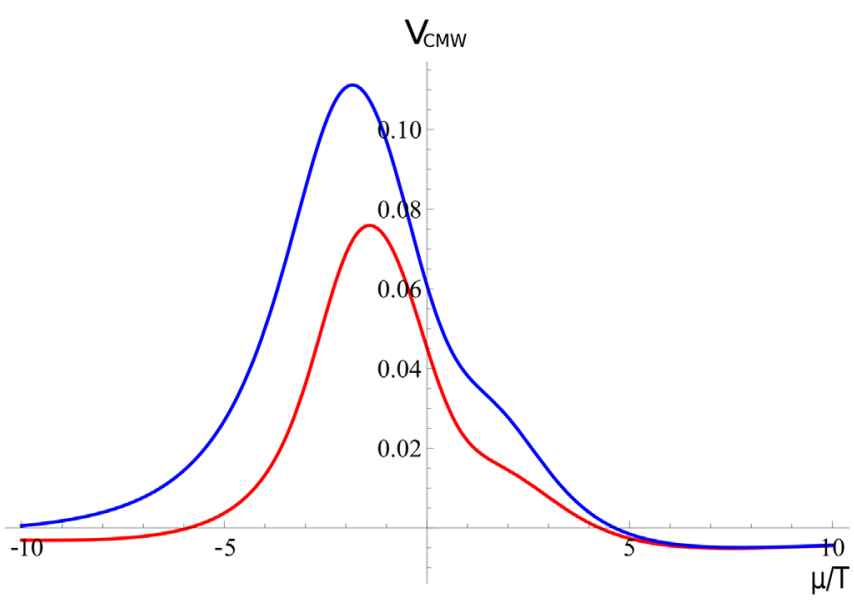

(a) Mode with plus sign in (76)

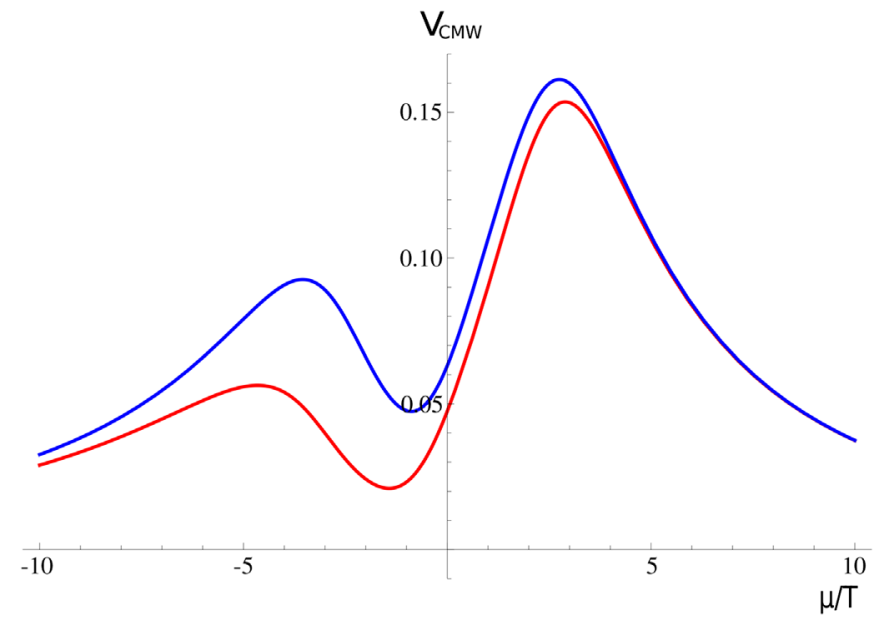

(b) Mode with minus sign in (76)

FIG. 3. Velocity of mixed CMVHW, $v_{C M V H W}$ (red), compared to the sum of the velocities of the individual waves $v_{C M H W}+v_{C V H W}$ (blue). In both panels $\frac{\mu_{5}}{T}=2$ and $\frac{B}{T^{2}}=\frac{\Omega}{T}=0.5$. We have used the equation of state (32).

In Eq. (74), $\left\{\mathbf{s}_{i}\right\}$ is the set of scalars made out of three independent vectors $\boldsymbol{k}, \boldsymbol{B}$, and $\boldsymbol{\Omega}$

$$
\begin{array}{ll}
\mathbf{s}_{1}=\boldsymbol{k}^{2}, & \\
\mathbf{s}_{2}=\boldsymbol{B}^{2}, & \mathbf{s}_{3}=\boldsymbol{B} \cdot \boldsymbol{k}, \\
\mathbf{s}_{4}=\boldsymbol{\Omega}^{2}, & \mathbf{s}_{5}=\boldsymbol{\Omega} \cdot \boldsymbol{k}, \\
\mathbf{s}_{6}=\boldsymbol{B} \cdot \boldsymbol{\Omega}, & \mathbf{s}_{7}=\boldsymbol{k} \cdot \boldsymbol{B} \times \boldsymbol{\Omega} .
\end{array}
$$

We have also defined a scalar $b$ (Appendix A 7), a vector $a_{j}$ (Appendix A 8), symmetric tensor $c_{j, k}$ (Appendix A 10), and tensor $d_{j, k, l}$ (Appendix A 9) in the seven-dimensional space generated by the above scalars (see Appendix A 7). All these objects are in terms of the components of the susceptibility matrix and the anomaly coefficients.

Due to difficulties in working with Eq. (74), from now on, we will focus on the special case wherein the magnetic field is parallel to the vorticity and study the propagation of waves along them. This case might be more relevant to the QCD fluid produced in heavy ion collisions. The dispersion relations of the modes in this case are as follows:

$$
\omega_{1,2}^{\boldsymbol{B} \Omega}=-\frac{\mathcal{A}_{1} B+\mathcal{A}_{3} \Omega \pm \sqrt{\left(\mathcal{A}_{1} B+\mathcal{A}_{3} \Omega\right)^{2}-\mathcal{E}\left(\mathcal{A}_{2} B^{2}+\mathcal{A}_{5} B \Omega+\mathcal{A}_{4} \Omega^{2}\right)}}{\mathcal{E}} k
$$

$$
\begin{gathered}
\omega_{3,4}^{\boldsymbol{B} \Omega}= \pm\left(\frac{n}{w} B+\Omega\right) \\
-\left\{\mathcal{C}\left(\mu \mu_{5}-\frac{n \mu_{5}}{3 w}\left(3 \mu^{2}+\mu_{5}^{2}\right)\right)-\mathcal{D} \frac{n \mu_{5}}{w} T^{2}\right\} \frac{B}{w} k \\
\omega_{5,6}^{\boldsymbol{B} \boldsymbol{Q}}= \pm k c_{s}
\end{gathered}
$$

What we observe in the scalar sector is the existence of two CMVHWs. In Fig. 3, we have plotted the velocities of these waves in two separate panels, the mode with a plus sign in panel a and the mode with a minus sign in panel $b$. In each panel we have also plotted with a blue curve the following quantity:

$$
v_{\text {sum }}:=v_{C M H W}+v_{C V H W}=\left.v_{C M V H W}\right|_{\mathbf{\Omega}=\mathbf{0}}+\left.v_{C M V H W}\right|_{\boldsymbol{B}=\mathbf{0}} .
$$

As the first point in the figure, the two CMVHWs neither always propagate in the same direction nor have the same velocities. More interestingly, one clearly sees that, in general,

$$
v_{C M V H W} \neq v_{\text {sum }} \text {. }
$$

It is simple to show that one of the situations in which $v_{C M V H W}$ equates with $v_{\text {sum }}$ is within the quark-gluon plasma produced in heavy ion collisions. In the latter case, $\mu_{5}=0$ and is equivalent to the limit $\frac{\mu}{\mu_{5}} \rightarrow \infty$. As it can be observed in Figure 3, in this limit $\frac{v_{C M V H W}}{v_{\text {sum }}} \rightarrow 1$. 
In the case of modes (77) and (78), one observes an interesting separation between the sound modes and the CAWs. CAWs in this case are pure and their propagation is also accompanied with two oppositely polarizing vortices.

Before ending this subsection let us emphasize that to our knowledge all of the results in this subsection are novel and have not been found in previous studies.

\section{PHENOMENOLOGY}

\section{A. Application to quark-gluon plasma}

In this part, we want to apply the new results found in this paper to a real QCD-type fluid case, namely the QCD fluid produced in heavy ion collision experiments. It has been understood that the quark-gluon plasma produced in a heavy ion collision is initially nonchiral, i.e., $\mu_{5}=0$. In this limit, we have $\beta_{3}=\alpha_{3}=0$ and the susceptibility matrix takes the following form:

$$
\tilde{\chi}=\left[\tilde{\chi}_{i j}\right]=\left[\begin{array}{ccc}
\frac{1}{c_{s}^{2} T}(w-\mu n) & \frac{n}{c_{s}^{2}} & 0 \\
\frac{1}{T}\left(\frac{n}{c_{s}^{2}}-\chi \mu\right) & \chi & 0 \\
0 & 0 & \chi
\end{array}\right] .
$$

As as result, the hydrodynamic modes given in (76), (77), and (78) simplify to

$\omega_{1,2}^{Q G P}= \pm \mathcal{C} \frac{B k}{\chi} \sqrt{\frac{\tilde{\chi}_{11} \tilde{\chi}_{22}}{\tilde{\chi}_{11} \tilde{\chi}_{22}-\tilde{\chi}_{12} \tilde{\chi}_{21}}} \pm \mathcal{C} \frac{\Omega \mu k}{\chi} \sqrt{\frac{\tilde{\chi}_{11}-\frac{\mathcal{D} T}{\mathcal{C} \mu} \tilde{\chi}_{12}}{\tilde{\chi}_{11} \tilde{\chi}_{22}-\tilde{\chi}_{12} \tilde{\chi}_{21}}}$

$$
\begin{gathered}
\omega_{3,4}^{Q G P}= \pm\left(\Omega_{L}+\Omega\right) \\
\omega_{5,6}^{Q G P}= \pm c_{s} k .
\end{gathered}
$$

Among the three equations given above, (81) is one of our main results regarding the QGP which deserves more explanation. To proceed, we first compute (79) for the current case, namely $v_{\text {sum }}^{Q G P}$. A simple calculation shows that $v_{\text {sum }}^{Q G P}$ is exactly the same as the velocity of mixed CMVHWs obtained from (81),

$$
v_{C M V H W}^{Q G P}=v_{\text {sum }}^{Q G P} .
$$

This result means that the $\mu_{5}=0$ case is an especial case in which CMHW and CVHW linearly mix to make CMVHWs; remember that we showed in general, when $\mu \neq 0$ and $\mu_{5} \neq 0$, this equality does not continue to hold.

Let us recall that the expressions given in front of the square roots in (81) are nothing but the familiar chiralmagnetic and chiral-vortical waves found in $[23,27]$ with the assumption of the energy and momentum perturbations being turned off. In [24,27], the induction of an electric quadrapole moment, or equivalently the observation of the difference between the elliptic flow of negative and positive charged hadrons in QGP, has been pointed out as the sign for the propagation of such waves, with the effect of CVWs being weaker than that of CMWs. Our computations show that even in the presence of energy and momentum perturbations the quadrapole moment would be induced too, while due to appearance of the square root expressions in (81), the effect might be predicted slightly differently compared to [24,27].

Another point with the hydrodynamic modes in QGP is that CAWs do not propagate in the plasma [see (82)], and the only propagating waves in the vector sector are two ordinary sound waves.

\section{B. Comments on dissipation in quark-gluon plasma}

In the whole of this paper up to now, we have focused on the propagation of waves in QCD-type chiral fluids in the absence of dissipation. Considering the dissipative effects makes the computations extremely complicated and it would be very hard to extract interesting physics from that. In this subsection, we study such effects in the case of the QGP fluid, with the simplifying point that there $\mu_{5}=0$. For more simplification, we neglect the effect of rotation in the plasma and just compute the hydrodynamic excitations in the magnetic field $B$. The set of six hydrodynamic modes are then given by

$$
\begin{aligned}
\omega_{1,2}^{Q G P-\text { diss }}= & \frac{-i \sigma k^{2}}{2 T}\left(\frac{T \tilde{\chi}_{11}-\mu \tilde{\chi}_{12}}{\tilde{\chi}_{11} \tilde{\chi}_{22}-\tilde{\chi}_{12} \tilde{\chi}_{21}}\right) \\
& \pm \sqrt{\left(\frac{\mathcal{C} B k}{\chi}\right)^{2} \frac{\tilde{\chi}_{11} \tilde{\chi}_{22}}{\tilde{\chi}_{11} \tilde{\chi}_{22}-\tilde{\chi}_{12} \tilde{\chi}_{21}}-\left(\frac{\sigma k^{2}}{2 T}\right)^{2}\left(\frac{T \tilde{\chi}_{11}+\mu \tilde{\chi}_{12}}{\tilde{\chi}_{11} \tilde{\chi}_{22}-\tilde{\chi}_{12} \tilde{\chi}_{21}}\right)^{2}-2 \frac{\mathcal{C B k}}{\chi} \frac{i \sigma_{5} k^{2}}{2 T}\left(\frac{T \tilde{\chi}_{11}+\mu \tilde{\chi}_{12}}{\tilde{\chi}_{11} \tilde{\chi}_{22}-\tilde{\chi}_{12} \tilde{\chi}_{21}}\right)}
\end{aligned}
$$

$$
\begin{gathered}
\omega_{3,4}^{Q G P-\text { diss }}= \pm \frac{n B}{w}-i \frac{k^{2} \eta+B^{2} \sigma}{w} \\
\omega_{5,6}^{Q G P-\text { diss }}= \pm c_{s} k-\frac{2 i k^{2} \eta}{3 w} .
\end{gathered}
$$

Obviously, the effect of the vector and chiral conductivities just appear in the first two modes. It is simple to see that when $\sigma=\sigma_{5}=0$, (85) gives the CMHWs in the first term of (81). These two modes are the CMHWs which dissipate due to the diffusion of vector and chiral charges. 
They are in fact dissipative CMHWs. The next two modes, namely $\omega_{3,4}^{Q G P-\text { diss }}$, are two oppositely circulating standing vortices which dissipate due to transverse shear effects as well as ohmic effects induced by the magnetic field. The same modes had been previously observed in [30] in a chiral fluid with just one single chirality. Finally, the last two modes (87) are the sound modes dissipating by the momentum diffusion in the transverse directions.

\section{CONCLUSION AND OUTLOOK}

As a main part of this paper we have found the hydrodynamic excitations in a fluid carrying both vector and axial charges. Neglecting the dissipative effects, none of these excitations are entropy producing; they are either adiabatic or anomalous waves in the fluid. In the latter case, the chiral transport may be observed in the fluid when fluid is coupled to an external magnetic field or is rotating around an axis.

In this paper, we have considered a general case in which the fluid is in the presence of a constant magnetic field $\boldsymbol{B}$ and simultaneously is rotating with a constant vorticity $\boldsymbol{\Omega}$. It has been shown that the full spectrum of the collective excitations constitute six modes in general; two of them are the coherent perturbations of the scalar currents, namely $J_{E}^{\mu}\left(=u_{\nu} T^{\nu \mu}\right), J^{\mu}, J_{5}^{\mu}$, while another four modes are made out of perturbations of all six scalar and vector currents.

The scalar modes are the mixture of CMHWs and CVHWs. There is an interesting point about Eq. (76). We have found that $v_{C M V H W}$ is actually a function of both $\mathcal{D}$ and $\mathcal{C}$. This suggests that by studying the effect of the chiral waves on the final spectrum of the charged particle in QGP, it might be possible to investigate the presence of gravitational anomalies. However, such observations require more precise experiments at higher energies compared to what currently is being done.

In the scalar-vector sector, we find four mixed sound-Alfvén-Coriolis modes which are all dispersive in general. When $\boldsymbol{\Omega}=0$, these modes become the mixed sound-Alfvén modes. While analogous $[29,30]$, we have used the terminology of Alfvén here although the Alfvén waves here are somewhat different from the standard Alfvén waves in magnetohydrodynamics. The main difference is that in the latter case the magnetic field has to be dynamical; in the former case, however, we have shown that chiral Alfvén waves propagate in the presence of an external constant magnetic field.

As mentioned above, the results in this paper have been found in the presence of a nondynamical magnetic field. It would be interesting to investigate how a dynamical magnetic field coupled to the flowing matter may affect the nature of the excitations [55]. To this end, one has to find the full spectrum of the chiral magnetohydrodynamics. We leave this issue for future studies.

It would be interesting to compute the full spectrum of the hydrodynamic modes in a QCD type fluid, microscopically. In the weak regime, using the recently developed chiral kinetic theory, one may extend the computations of [32] to the case in which the axial and vector charge fluctuations are coupled to energy and momentum fluctuations. It should be noted that the chiral kinetic theory computations are basically done in the Laboratory frame. It would be interesting to compare the results of the current paper in the Landau-Lifshitz frame with the results of the Laboratory frame.

In another direction, it would be of more interest to find the spectrum of the hydrodynamic excitations propagating on top of the expanding quark-gluon plasma [59]. Recently, the authors of [61] have studied the linear fluctuations around a Bjorken flow analytically although they neither coupled the fluid to the magnetic field nor considered the chiral transport in their work. It would be phenomenologically important to extend the subject of [61] to the chiral QCD case.

Apart from the quark-gluon plasma, our results found in this paper may be applied to other phenomena in physics as well. A different place to explore is indeed the neutrino matter at the core of the supernova star, wherein a gas of noninteracting fermions is flowing [62]. It would be interesting to see how the velocities of the hydrodynamic waves change with the density there. We leave further study on the issue to our future work.

\section{ACKNOWLEDGMENTS}

We would like to thank Prof. Mohsen Alishahiha for encouragement and supporting the Larak-Particle-Pheno group. We would also like to thank M. Mohammadi Najafaabdi for reading the paper thoroughly and giving useful comments. We thank A. Akhavan. N. A. would like to thank Prof. H. Arfaei for illuminating discussions on gravitational anomalies. A. D. would like to thank P. V. Buividovich and S. N. Valgushev for discussions. The work of A. D. was supported by the S. Kowalevskaja award from the Alexander von Humboldt Foundation. We would like to thank Maxim Chernodub for discussions.

\section{APPENDIX: SUSCEPTIBILITY MATRIX AND ANOMALY COEFFICIENTS}

\section{Transforming from one thermodynamic basis to another}

Using the following thermodynamic relations, one can express the hydrodynamic modes in terms of the coherent excitations of a more physical set of variables, namely $\left\{\delta \epsilon, \delta n, \delta n_{5}\right\}$,

$$
\delta T=\left(\frac{\partial T}{\partial \epsilon}\right) \delta \epsilon+\left(\frac{\partial T}{\partial n}\right) \delta n+\left(\frac{\partial T}{\partial n_{5}}\right) \delta n_{5}
$$




$$
\begin{array}{r}
\delta \mu=\left(\frac{\partial \mu}{\partial \epsilon}\right) \delta \epsilon+\left(\frac{\partial \mu}{\partial n}\right) \delta n+\left(\frac{\partial \mu}{\partial n_{5}}\right) \delta n_{5} \\
\delta \mu_{5}=\left(\frac{\partial \mu_{5}}{\partial \epsilon}\right) \delta \epsilon+\left(\frac{\partial \mu_{5}}{\partial n}\right) \delta n+\left(\frac{\partial \mu_{5}}{\partial n_{5}}\right) \delta n_{5} .
\end{array}
$$

\section{Susceptibility Matrix and the Constraint Relations}

In order to express the dynamical fields $\epsilon, n$, and $n_{5}$ in terms of the variables (9), we consider the susceptibility matrix as

$$
\tilde{\chi}=\left[\begin{array}{lll}
\alpha_{1}=\frac{\partial \epsilon}{\partial T}, & \alpha_{2}=\frac{\partial \epsilon}{\partial \mu}, & \alpha_{3}=\frac{\partial \epsilon}{\partial \mu_{5}} \\
\beta_{1}=\frac{\partial n}{\partial T}, & \beta_{2}=\frac{\partial n}{\partial \mu}, & \beta_{3}=\frac{\partial n}{\partial \mu_{5}} \\
\gamma_{1}=\frac{\partial n_{5}}{\partial T}, & \gamma_{2}=\frac{\partial n_{5}}{\partial \mu}, & \gamma_{3}=\frac{\partial n_{5}}{\partial \mu_{5}}
\end{array}\right] .
$$

Let us recall that the elements of this matrix are not generally independent; using the thermodynamic relations, one simply shows that

$$
\begin{gathered}
\beta_{1}=\frac{1}{c_{s}^{2}} \frac{n}{T}-\beta_{2} \frac{\mu}{T}-\gamma_{2} \frac{\mu_{5}}{T} \\
\beta_{3}=\gamma_{2} \\
\gamma_{1}=\frac{1}{c_{s}^{2}} \frac{n_{5}}{T}-\beta_{3} \frac{\mu}{T}-\gamma_{3} \frac{\mu_{5}}{T} .
\end{gathered}
$$

\section{Matrix $M_{a b}^{B \Omega}$}

The matrix $M_{a b}^{B \Omega}$ is given by

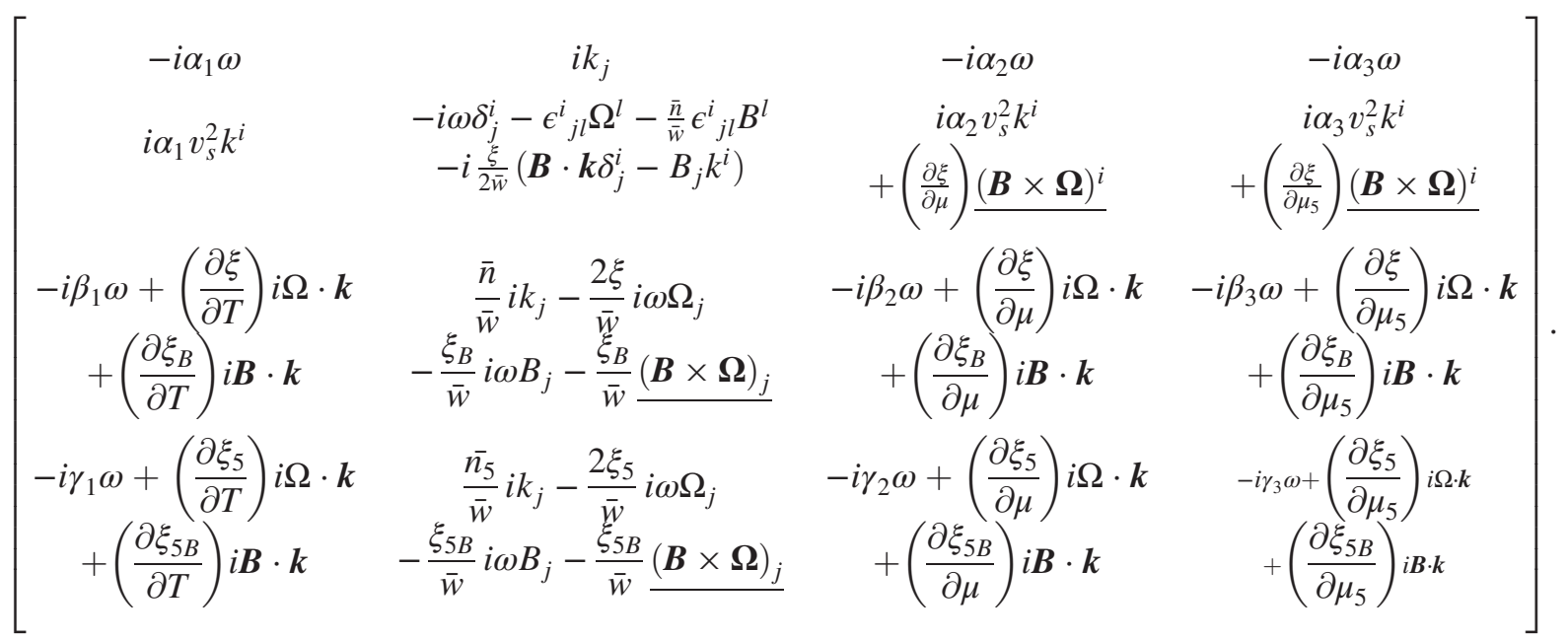

An interesting point with this matrix is the appearance of the terms including both vorticity and the magnetic field. However, these terms disappear when the magnetic field is parallel to the vorticity.

\section{4. $x_{1}, x_{2}$ coefficients}

The anomaly coefficients in the structure of $\mathcal{A}_{3}$ and $\mathcal{A}_{4}$ in [(57)] are given by the following five expressions. The first two, namely $x_{1}$ and $x_{2}$, are in the structure of $\mathcal{A}_{3}$ :

\begin{tabular}{lc}
\hline \hline Coefficient & Structure \\
\hline$x_{1}$ & $T\left(\alpha_{[2} \beta_{3]}+\frac{T n_{5}}{w} \alpha_{[2} \beta_{1]}-\frac{T n}{w} \alpha_{[2} \gamma_{1]}+\frac{n_{5} \mu_{5}}{w} \alpha_{[3} \beta_{2]}-\frac{n \mu_{5}}{w} \alpha_{[3} \gamma_{2]}+\frac{2 \mu_{5} T}{w}\right)$ \\
$x_{2}$ & $\frac{1}{w}\left(n_{5} \alpha_{[2} \beta_{1]}-n \alpha_{[2} \gamma_{1]}\right)\left(\mu^{2}+\mu_{5}^{2}\right)+\frac{2 \mu \mu_{5}}{w}\left(n_{5} \alpha_{[1} \beta_{3]}-n \alpha_{[1} \gamma_{3]}\right)$ \\
& $-\mu\left(\alpha_{[1} \beta_{3]}+\alpha_{[1} \gamma_{2]}\right)+\mu_{5}\left(\alpha_{[1} \beta_{2]}+\alpha_{[1} \gamma_{3]}\right)+\frac{2 \mu_{5}}{w}\left(\mu^{2}+\frac{\mu_{5}^{2}}{3}\right) \mathcal{E}$ \\
\hline
\end{tabular}




\section{5. $y_{1}, y_{2}, y_{3}$ coefficients}

The anomaly coefficients in the structure of $\mathcal{A}_{4}$ in (57) are $y_{1}, y_{2}$, and $y_{3}$,

\begin{tabular}{ll}
\hline \hline Coefficient & Structure \\
\hline$y_{1}$ & $4 \alpha_{1} \mu^{2}\left(1-\frac{\mu n}{w}\right)-4 \alpha_{1} \mu_{5}^{2}\left(1-\frac{\mu_{5} n_{5}}{w}\right)+\frac{4 \alpha_{1} \mu \mu_{5}}{w}\left(\mu_{5} n-\mu n_{5}\right)$ \\
& $-\frac{4\left(\alpha_{[1} \beta_{3]}+\alpha_{[1} \gamma_{2]}\right] \mu \mu_{5}}{w}\left(\mu^{2}+\frac{\mu_{5}^{2}}{3}\right)+\frac{4\left(\alpha_{[1} \beta_{2]}+\alpha_{[1} \gamma_{3]}\right) \mu_{5}^{2}}{w}\left(\mu^{2}+\frac{\mu_{5}^{2}}{3}\right)$ \\
& $+\left(\alpha_{[2} \beta_{1]} n_{5}-\alpha_{[2} \gamma_{1]} n\right) \frac{16 \mu^{2} \mu_{5}^{3}}{3 w^{2}}+2\left(\alpha_{[1} \beta_{3]} n_{5}-\alpha_{[1} \gamma_{3]} n\right) \frac{4 \mu \mu_{5}^{2}}{w^{2}}\left(\mu^{2}+\frac{\mu_{5}^{2}}{3}\right)$ \\
& $+\left(\alpha_{[2} \beta_{1]} n_{5}-\alpha_{[2} \gamma_{1]} n\right) \frac{4 \mu_{5}}{w^{2}}\left(\mu^{4}+\frac{\mu_{5}^{4}}{3}\right)+\frac{4 \mu_{5}^{2}}{w^{2}}\left(\mu^{2}+\frac{\mu_{5}^{2}}{3}\right)^{2} \mathcal{E}$ \\
& $4 T^{3}\left(\frac{\alpha_{2} n}{w}+\left(\alpha_{[1} \gamma_{2]} n-\alpha_{[1} \beta_{2]} n_{5}\right) \frac{\mu_{5} T}{3 w^{2}}+2\left(\alpha_{[2} \gamma_{3} n-\alpha_{[2} \beta_{3]} n_{5}\right) \frac{\mu_{5}^{2}}{w^{2}}+\alpha_{[2} \beta_{3]} \frac{\mu_{5}}{w}+\frac{\mu_{5}^{2} T}{w^{2}} \mathcal{E}\right)$ \\
& $4 T\left(\left(\alpha_{3} \mu_{5}-\alpha_{2} \mu\right)\left(1-\frac{2 \mu_{5} n_{5}}{w}\right)+\alpha_{1}\left(\mu_{5} n_{5}-\mu n\right) \frac{T}{w}+\alpha_{2}\left(\mu^{2}-\mu_{5}^{2}\right) \frac{n}{w}\right.$ \\
$y_{3}$ & $+\left(\alpha_{[2} \beta_{3]} \frac{\mu_{5}}{w}+\frac{2 \mu_{5}^{2} n}{w^{2}} \alpha_{[2} \gamma_{3]}-\frac{2 \mu_{5}^{2} n_{5}}{w^{2}} \alpha_{[2} \beta_{3]}\right)\left(\mu^{2}+\frac{\mu_{5}^{2}}{3}\right)+\left(\alpha_{[1} \gamma_{2]} n-\alpha_{[1} \beta_{2]} n_{5}\right) \frac{4 \mu^{3} T}{3 w^{2}}$ \\
& $+\left(\left(\alpha_{[1} \beta_{3]} \mu_{5}-\alpha_{[1} \beta_{2]} \mu\right) n_{5}+\left(\alpha_{[1} \gamma_{2]} \mu-\alpha_{[1} \gamma_{3]} \mu_{5}\right) n\right) \frac{2 \mu \mu_{5} T}{w^{2}}$ \\
& $\left.+\left(\left(\alpha_{[1} \beta_{2]} \mu_{5}-\alpha_{[1} \gamma_{2]} \mu\right)+\left(\alpha_{[1} \gamma_{3]} \mu_{5}-\alpha_{[1} \beta_{3]} \mu\right)\right) \frac{\mu_{5} T}{w}+\frac{2 \mu_{5}^{2} T}{w^{2}}\left(\mu^{2}+\frac{\mu_{5}^{2}}{3}\right) \mathcal{E}\right)$
\end{tabular}

\section{6. $z_{1}, z_{2}$ coefficients}

The anomaly coefficients in the structure of $\mathcal{A}_{5}$ in (72), namely $z_{1}$ and $z_{2}$, are given by

\begin{tabular}{lc}
\hline \hline Coefficient & Structure \\
\hline$z_{1}$ & $2\left(3 \mu^{2} \mu_{5}^{2}+\frac{1}{3} \mu_{5}^{4}\right)\left(n_{5} \alpha_{[1} \beta_{3]}-n \alpha_{[1} \gamma_{3]}\right)-2\left(\frac{4}{3} \mu \mu_{5}^{3}+2 \mu^{3} \mu_{5}\right)\left(n_{5} \alpha_{[1} \beta_{2]}-n \alpha_{[1} \gamma_{2]}\right)$ \\
& $-2 w\left(\mu^{2} \mu_{5}+\frac{1}{3} \mu_{5}^{3}\right)\left(\alpha_{[1} \beta_{3]}+\alpha_{[1} \gamma_{2]}\right)+2 \mu \mu_{5}^{2} w\left(\alpha_{[1} \beta_{2]}+\alpha_{[1} \gamma_{3]}\right)+4 \mu \mu_{5}^{2}\left(\mu^{2}+\frac{\mu_{5}^{2}}{3}\right) \mathcal{E}$ \\
& $-4 \alpha_{1} \mu w\left(\mu n+\mu_{5} n_{5}-w\right)$ \\
& $-4 \mu \mu_{5}^{2}\left(n_{5} \alpha_{[2} \beta_{3]}-n \alpha_{[2} \gamma_{3]}\right)+2 \mu \mu_{5} w \alpha_{[2} \gamma_{3]}$ \\
$z_{2}$ & $+2 \mu_{5}^{2}\left(n_{5} \alpha_{[1} \beta_{3]}-n \alpha_{[1} \gamma_{3]}\right)-2 \mu_{5} T w\left(\alpha_{[1} \beta_{3]}+\alpha_{[1} \gamma_{2]}\right)-4 \mu \mu_{5} n_{5} T\left(n_{5} \alpha_{[1} \beta_{2]}-n \alpha_{[1} \gamma_{2]}\right)$ \\
& $-2\left(\alpha_{1} n T-\alpha_{2}\left(\mu n+2 \mu_{5} n_{5}-w\right)-\alpha_{3} \mu_{5} n\right) w+4 \mu \mu_{5}^{2} T \mathcal{E}$
\end{tabular}

\section{7. $b$ coefficient}

The only scalar coefficient in (74) is $b$ which given by

\begin{tabular}{rl}
\hline \hline$b$ & $2 \mathcal{C}\left\{\frac{1}{2}\left(\mu_{5} \beta_{[1} \gamma_{3]}-\mu \beta_{[1} \gamma_{2]}\right)+\frac{n}{2 w}\left(\beta_{[1} \gamma_{2]}-\frac{n}{w} \alpha_{[1} \gamma_{2]}+\frac{n_{5}}{w} \alpha_{[1} \beta_{2]}\right)\left(\mu^{2}+\mu_{5}^{2}\right)+\frac{\mu}{2 w}\left(n \alpha_{[1} \gamma_{2]}-n_{5} \alpha_{[1} \beta_{2]}\right)\right.$ \\
& $\left.+\frac{\mu_{5}}{w}\left(\frac{\mu n}{w}-\frac{1}{2}\right)\left(n \alpha_{[1} \gamma_{3]}-n_{5} \alpha_{[1} \beta_{3]}\right)-\frac{\mu \mu_{5} n}{w} \beta_{[1} \gamma_{3]}+\frac{\mu_{5} n}{2 w^{2}}\left(c_{s}^{2}-\frac{1}{2}\right)\left(\mu^{2}+\frac{\mu_{5}^{2}}{3}\right) \mathcal{E}+\frac{\mu \mu_{5}}{4 w} \mathcal{E}\right\}$ \\
& $2 \mathcal{D} T\left\{\frac{n T}{2 w}\left(\beta_{[1} \gamma_{2]}-\frac{n}{w} \alpha_{[1} \gamma_{2]}+\frac{n_{5}}{w} \alpha_{[1} \beta_{2]}\right)+\frac{n \mu_{5}}{w}\left(\beta_{[2} \gamma_{3]}-\frac{n}{w} \alpha_{[2} \gamma_{3]}+\frac{n_{5}}{w} \alpha_{[2} \beta_{3]}\right)+\frac{\mu_{5} n T}{2 w^{2}}\left(c_{s}^{2}-\frac{1}{2}\right) \mathcal{E}\right\}$
\end{tabular}




\section{8. $a_{j}$ coefficients}

The non-vinishing components of the vector $a_{j}$ in (74) are $a_{3}$ and $a_{5}$ as the following:

\begin{tabular}{ll}
\hline \hline Coefficient & Structure \\
\hline$a_{3}$ & $\mathcal{C}\left\{\frac{1}{2}\left(\alpha_{[3} \beta_{1]}+\alpha_{[2} \gamma_{1]}\right)+\left(\frac{\mu_{5} n_{5}}{2 w} \alpha_{[1} \beta_{3]}-\frac{\mu n_{5}}{2 w} \alpha_{[1} \beta_{2]}+\frac{\mu n}{2 w} \alpha_{[1} \gamma_{2]}-\frac{\mu_{5} n}{2 w} \alpha_{[1} \gamma_{3]}\right)\right.$ \\
& $\left.-\frac{\mu_{5} n}{w^{2}}\left(\mu^{2}+\frac{\mu_{5}^{2}}{3}\right) \mathcal{E}+\frac{6 \mu \mu_{5}}{w} \mathcal{E}\right\}-\mathcal{D}\left\{\frac{\mu_{5} n T^{2}}{w^{2}}\right\} \mathcal{E}$ \\
& $2 \mathcal{C}\left\{\frac{1}{2}\left(-\mu \alpha_{[1} \beta_{3]}+\mu_{5} \alpha_{[1} \beta_{2]}-\mu \alpha_{[1} \gamma_{2]}+\mu_{5} \alpha_{[1} \gamma_{3]}\right)+\frac{\mu_{5}}{w}\left(\mu^{2}+\frac{\mu_{5}^{2}}{3}\right) \mathcal{E}\right.$ \\
& $\left.+\left(\alpha_{[1} \gamma_{2]} \frac{n}{2 w}-\alpha_{[1} \beta_{2]} \frac{n_{5}}{2 w}\right)\left(\mu^{2}+\mu_{5}^{2}\right)+\left(\alpha_{[1} \beta_{3]}-\alpha_{[1} \gamma_{3]}\right) \frac{\mu \mu_{5} n_{5}}{w}\right\}$ \\
& $+2 \mathcal{D} T\left\{\frac{1}{2} \alpha_{[2} \beta_{3]}+\frac{\mu_{5}}{w}\left(\alpha_{[2} \gamma_{3]} n-\alpha_{[2} \beta_{3]} n_{5}\right)+\frac{T}{2 w}\left(n \alpha_{[1} \gamma_{2]}-n_{5} \alpha_{[1} \beta_{2]}\right)+\frac{\mu_{5} T}{w} \mathcal{E}\right\}$ \\
\hline
\end{tabular}

\section{9. $d_{j, k, l}$ coefficients}

The tensor $d_{j, k, l}$ in (74) is a fully symmetric rank-3 tensor with the following nonvanishing components:

\begin{tabular}{ll}
\hline \hline Coefficient & Structure \\
\hline$d_{3,3,3}$ & $\mathcal{C}\left\{\frac{c_{s}^{2} n^{2}}{2 w^{3}}\left(\mu n \alpha_{[1} \gamma_{2]}-\mu_{5} n \alpha_{[1} \gamma_{3]}-\mu n_{5} \alpha_{[1} \beta_{2]}+\mu_{5} n_{5} \alpha_{[1} \beta_{3]}\right)-\frac{c_{s}^{2} n^{2}}{2 w^{2}}\left(\alpha_{[1} \beta_{3]}+\alpha_{[1} \gamma_{2]}\right)+\frac{c_{s}^{2} \mu \mu_{5} n^{2}}{w^{3}} \mathcal{E}\right\}$ \\
& $\mathcal{C}\left\{c_{s}^{2} \mu_{5}\left(\alpha_{[1} \beta_{2]}+\alpha_{[1} \gamma_{3]}\right)-c_{s}^{2} \mu\left(\alpha_{[1} \beta_{3]}+\alpha_{[1} \gamma_{2]}\right)+\frac{2 c_{s}^{2} \mu_{5}}{w}\left(\mu^{2}+\frac{\mu_{5}^{2}}{3}\right)\right.$ \\
& $\left.-\frac{c_{s}^{2}}{w}\left(n_{5} \alpha_{[1} \beta_{2]}-n \alpha_{[1} \gamma_{2]}\right)\left(\mu^{2}+\mu_{5}^{2}\right)+\frac{2 c_{s}^{2} \mu \mu_{5}}{w}\left(n_{5} \alpha_{[1} \beta_{3]}-n \alpha_{[1} \gamma_{3]}\right)\right\}$ \\
& $+\mathcal{D} T\left\{-\frac{c_{s}^{2} T}{w}\left(n_{5} \alpha_{[1} \beta_{2]}-n \alpha_{[1} \gamma_{2]}\right)-\frac{2 c_{s}^{2} \mu_{5}}{w}\left(n_{5} \alpha_{[2} \beta_{3]}-n \alpha_{[2} \gamma_{3]}\right)+c_{s}^{2} \alpha_{[2} \beta_{3]}+\frac{2 c_{s}^{2} \mu_{5} T}{w} \mathcal{E}\right\}$ \\
& $\mathcal{C}\left\{-\frac{c_{s}^{2} n^{2}}{w^{3}}\left(n_{5} \alpha_{[1} \beta_{2]}-n \alpha_{[1} \gamma_{2]}\right)\left(\mu^{2}+\mu_{5}^{2}\right)+\frac{2 c_{s}^{2} \mu \mu_{5} n^{2}}{w^{3}}\left(n_{5} \alpha_{[1} \beta_{3]}-n \alpha_{[1} \gamma_{3]}\right)\right.$ \\
$d_{3,3,5} \quad$ & $+\frac{c_{s}^{2} n^{2}}{2 w^{2}}\left(\mu_{5}\left(\alpha_{[1} \beta_{2]}-\alpha_{[1} \gamma_{3]}\right)-\mu\left(\alpha_{[1} \beta_{3]}-\alpha_{[1} \gamma_{2]}\right)\right)+\frac{2 c_{s}^{2} \mu_{5} n^{2}}{w^{3}}\left(\mu^{2}+\frac{\mu_{5}^{2}}{3}\right) \mathcal{E}$ \\
$=d_{5,3,3}$ & $\left.+\frac{c_{s}^{2} n_{5} n}{w^{2}}\left(\mu_{5} \alpha_{[1} \beta_{3]}-\mu \alpha_{[1} \beta_{2]}\right)-\frac{c_{s}^{2} n}{w}\left(\alpha_{[1} \beta_{3]}+\alpha_{[1} \gamma_{2]}\right)+\frac{2 c_{s}^{2} \mu \mu_{5} n}{w^{2}} \mathcal{E}\right\}$ \\
& $-\mathcal{D} T\left\{\frac{c_{s}^{2} n^{2} T}{w^{3}}\left(\left(n_{5} \alpha_{[1} \beta_{2]}-n \alpha_{[1} \gamma_{2]}\right)+4 \mu_{5}\left(n_{5} \alpha_{[2} \beta_{3]}-n \alpha_{[2} \gamma_{3]}\right)\right)-\frac{2 c_{s}^{2} n^{2}}{w^{2}} \alpha_{[2} \beta_{3]}-\frac{c_{s}^{2} \mu_{5} n^{2} T^{2}}{w^{3}} \mathcal{E}\right\}$.
\end{tabular}

10. $c_{j, k}$ coefficients

The tensor $c_{j, k}$ in (74) is a symmetric tensor with the following nonvanishing components:

\begin{tabular}{lc}
\hline \hline Coefficient & Structure \\
\hline$c_{3,1}=c_{1,3}$ & $\mathcal{C}\left\{\frac{c_{s}^{2}}{2}\left(\alpha_{[1} \beta_{3]}+\alpha_{[1} \gamma_{2]}\right)-\frac{c_{s}^{2}}{2 w}\left(\mu n \alpha_{[1} \gamma_{2]}-\mu_{5} n \alpha_{[1} \gamma_{3]}-\mu n_{5} \alpha_{[1} \beta_{2]}+\mu_{5} n_{5} \alpha_{[1} \beta_{3]}\right)\right.$ \\
& $\left.+\frac{c_{s}^{2} \mu_{5} n}{w^{2}}\left(\mu^{2}+\frac{\mu_{5}^{2}}{3}\right) \mathcal{E}-\frac{3 c_{s}^{2} \mu \mu_{5}}{w} \mathcal{E}\right\}+\mathcal{D} T\left\{\frac{c_{s}^{2} \mu_{5} n T^{2}}{w^{2}} \mathcal{E}\right\}$ \\
$c_{5,2}=c_{2,5}$ & $2 \mathcal{C}\left\{\frac{n}{2 w^{2}}\left(\left(\mu n-\mu_{5} n_{5}\right) \alpha_{[1} \beta_{3]}+\left(\mu n_{5}-\mu_{5} n\right) \alpha_{[1} \beta_{2]}\right)-\frac{n^{2}}{2 w^{2}} \beta_{[1} \gamma_{2]}\left(\mu^{2}+\mu_{5}^{2}\right)+\frac{\mu n}{2 w} \beta_{[1} \gamma_{2]}\right.$ \\
\hline
\end{tabular}




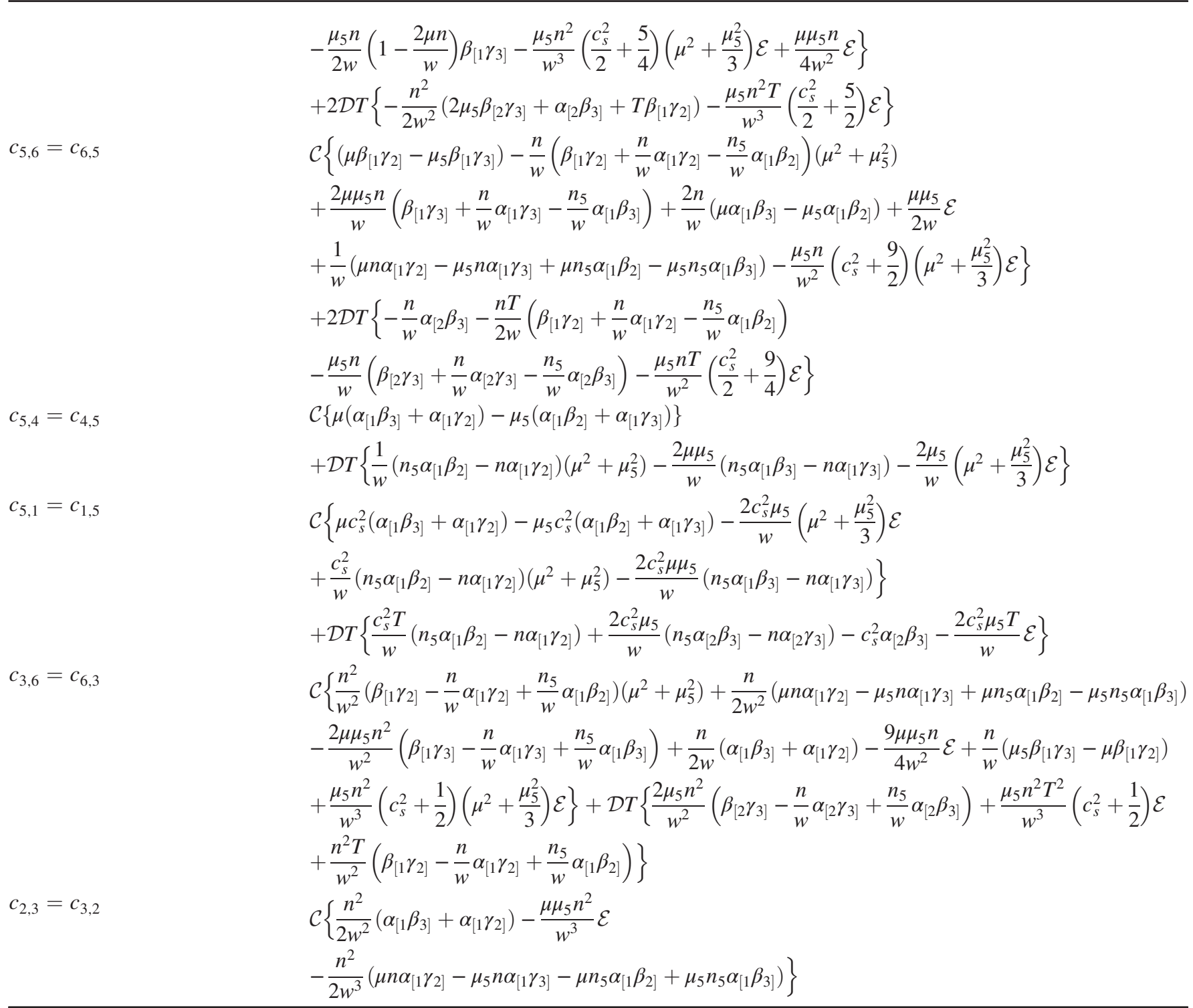

[1] A. Vilenkin, Equilibrium parity violating current in a magnetic field, Phys. Rev. D 22, 3080 (1980).

[2] A. Vilenkin, Macroscopic parity violating effects: Neutrino fluxes from rotating black holes and in rotating thermal radiation, Phys. Rev. D 20, 1807 (1979).

[3] D. T. Son and N. Yamamoto, Berry Curvature, Triangle Anomalies, and the Chiral Magnetic Effect in Fermi Liquids, Phys. Rev. Lett. 109, 181602 (2012).

[4] M. A. Stephanov and Y. Yin, Chiral Kinetic Theory, Phys. Rev. Lett. 109, 162001 (2012).
[5] J.-W. Chen, S. Pu, Q. Wang, and X.-N. Wang, Berry Curvature and 4-Dimensional Monopole in Relativistic Chiral Kinetic Equation, Phys. Rev. Lett. 110, 262301 (2013).

[6] P. V. Buividovich, M. N. Chernodub, E. V. Luschevskaya, and M. I. Polikarpov, Numerical evidence of chiral magnetic effect in lattice gauge theory, Phys. Rev. D 80, 054503 (2009).

[7] P. V. Buividovich, M. N. Chernodub, D. E. Kharzeev, T. Kalaydzhyan, E. V. Luschevskaya, and M. I. Polikarpov, 
Magnetic-Field-Induced Insulator-Conductor Transition in SU(2) Quenched Lattice Gauge Theory, Phys. Rev. Lett. 105, 132001 (2010).

[8] M. Puhr and P. V. Buividovich, A Numerical Study of Nonperturbative Corrections to the Chiral Separation Effect in Quenched Finite-Density QCD, Phys. Rev. Lett. 118, 192003 (2017).

[9] P. V. Buividovich and S. N. Valgushev, Proc. Sci., LATTICE2016 (2016) 253 [arXiv:1611.05294].

[10] J. Erdmenger, M. Haack, M. Kaminski, and A. Yarom, Fluid dynamics of R-charged black holes, J. High Energy Phys. 01 (2009) 055.

[11] N. Banerjee, J. Bhattacharya, S. Bhattacharyya, S. Dutta, R. Loganayagam, and P. Surowka, Hydrodynamics from charged black branes, J. High Energy Phys. 01 (2011) 094.

[12] D. T. Son and P. Surowka, Hydrodynamics with Triangle Anomalies, Phys. Rev. Lett. 103 (2009) 191601.

[13] D. E. Kharzeev and H. U. Yee, Anomalies and time reversal invariance in relativistic hydrodynamics: Second order and higher dimensional formulations, Phys. Rev. D 84 (2011) 045025

[14] S. Sen and N. Yamamoto, Chiral Shock Waves, Phys. Rev. Lett. 118, 181601 (2017).

[15] N. Yamamoto, Chiral transport of neutrinos in supernova, EPJ Web Conf. 137, 09013 (2017).

[16] M. Kaminski, C. F. Uhlemann, M. Bleicher, and J. SchaffnerBielich, Anomalous hydrodynamics kicks neutron stars, Phys. Lett. B 760, 170 (2016).

[17] M. Giovannini and M. E. Shaposhnikov, Primordial hypermagnetic fields and triangle anomaly, Phys. Rev. D 57, 2186 (1998).

[18] F. M. D. Pellegrino, M. I. Katsnelson, and M. Polini, Helicons in the Weyl semimetals, Phys. Rev. B 92, 201407(R) (2015).

[19] K. Landsteiner, Anomalous transport of Weyl fermions in Weyl semimetals, Phys. Rev. B 89, 075124 (2014).

[20] K. Landsteiner, Notes on anomaly induced transport, Acta Phys. Pol. B 47, 2617 (2016).

[21] D. T. Son and A. R. Zhitnitsky, Quantum anomalies in dense matter, Phys. Rev. D 70, 074018 (2004).

[22] K. Fukushima, D. E. Kharzeev, and H. J. Warringa, Chiral magnetic effect, Phys. Rev. D 78, 074033 (2008).

[23] D. E. Kharzeev and H. U. Yee, Chiral magnetic wave, Phys. Rev. D 83, 085007 (2011).

[24] Y. Burnier, D. E. Kharzeev, J. Liao, and H. U. Yee, Chiral Magnetic Wave at Finite Baryon Density and the Electric Quadrupole Moment of Quark-Gluon Plasma in Heavy Ion Collisions, Phys. Rev. Lett. 107, 052303 (2011).

[25] L. Adamczyk et al. (STAR Collaboration), Observation of Charge Asymmetry Dependence of Pion Elliptic Flow and the Possible Chiral Magnetic Wave in Heavy-Ion Collisions, Phys. Rev. Lett. 114, 252302 (2015).

[26] R. Belmont (ALICE Collaboration), Charge-dependent anisotropic flow studies and the search for the Chiral Magnetic Wave in ALICE, Nucl. Phys. A931, 981 (2014).

[27] Y. Jiang, X. G. Huang, and J. Liao, Chiral vortical wave and induced flavor charge transport in a rotating quark-gluon plasma, Phys. Rev. D 92, 071501 (2015).

[28] M. Stephanov, H. U. Yee, and Y. Yin, Collective modes of chiral kinetic theory in a magnetic field, Phys. Rev. D 91, 125014 (2015).
[29] N. Yamamoto, Chiral Alfvén Wave in Anomalous Hydrodynamics, Phys. Rev. Lett. 115, 141601 (2015).

[30] N. Abbasi, A. Davody, K. Hejazi, and Z. Rezaei, Hydrodynamic waves in an anomalous charged fluid, Phys. Lett. B 762, 23 (2016).

[31] M. N. Chernodub, Chiral heat wave and mixing of magnetic, vortical and heat waves in chiral media, J. High Energy Phys. 01 (2016) 100.

[32] D. Frenklakh, Chiral heat wave and mixed waves in kinetic theory, Phys. Rev. D 94, 116010 (2016).

[33] L. D. Landau and E. M. Lifshitz, Fluid Mechanics (Pergamon, New York, 1987).

[34] J. Bhattacharya, S. Bhattacharyya, S. Minwalla, and A. Yarom, A Theory of first order dissipative superfluid dynamics, J. High Energy Phys. 05 (2014) 147.

[35] Y. Neiman and Y. Oz, Relativistic hydrodynamics with general anomalous charges, J. High Energy Phys. 03 (2011) 023.

[36] J. H. Gao, Z. T. Liang, S. Pu, Q. Wang, and X. N. Wang, Chiral Anomaly and Local Polarization Effect from the Quantum Kinetic Approach, Phys. Rev. Lett. 109, 232301 (2012).

[37] K. Landsteiner, E. Megias, and F. Pena-Benitez, Gravitational Anomaly and Transport, Phys. Rev. Lett. 107, 021601 (2011).

[38] A. V. Sadofyev and M. V. Isachenkov, The Chiral magnetic effect in hydrodynamical approach, Phys. Lett. B 697, 404 (2011).

[39] P. Kovtun, Lectures on hydrodynamic fluctuations in relativistic theories, J. Phys. A 45, 473001 (2012).

[40] N. Abbasi and A. Davody, Dissipative charged fluid in a magnetic field, Phys. Lett. B 756, 161 (2016).

[41] J. Casalderrey-Solana, H. Liu, D. Mateos, K. Rajagopal, and U. A. Wiedemann, Gauge/String Duality, Hot QCD and Heavy Ion Collisions (Cambridge University Press, Cambridge, England, 2014).

[42] We use the metric $g_{\mu \nu}=(-1,1,1,1)$ in this paper.

[43] X. G. Huang and J. Liao, Axial Current Generation from Electric Field: Chiral Electric Separation Effect, Phys. Rev. Lett. 110, 232302 (2013).

[44] Throughout this paper wherever we mention scalar or vector, we mean the representations of the $S O(3)$ spatial rotational group.

[45] $A_{[i} B_{j]}=A_{i} B_{j}-A_{j} B_{i}$.

[46] We work in a system of units where $c=1$.

[47] It can be simply obtained by solving $\partial_{\nu} v_{C M H W}=0$ with $\nu=\mu / T$.

[48] Some part of this difference might be related to the difference in the choice of frame between our paper and [13]. We will shed light on the issue in [49].

[49] N. Abbasi, K. Naderi, and F. Taghinavaz (unpublished).

[50] D. D. Schnack, Lectures in Magnetohydrodynamics (Springer-Verlag, Berlin, 2009).

[51] We would like to thank to referee for pointing out the true name of these modes to us.

[52] T. Kalaydzhyan and E. Murchikova, Thermal chiral vortical and magnetic waves: New excitation modes in chiral fluids, Nucl. Phys. B919, 173 (2017).

[53] Note that the idea of studying the momentum perturbations was first in [29], and then the authors of [30] took into account the energy perturbations as well. 
[54] These modes differ from chiral vortical waves found in [27]. In the latter reference, the scalar perturbations in a nonchiral limit $\left(\mu_{5}=0\right)$ have been found when the temperature is kept fixed.

[55] See [56-58] for recent studies.

[56] M. Giovannini, Anomalous magnetohydrodynamics, Phys. Rev. D 88, 063536 (2013).

[57] A. K. Pandey, A study on the collective behavior of chiral plasma using first and second order conformal hydrodynamics, arXiv:1609.01848.

[58] N. Sadooghi and S. M. A. Tabatabaee, The effect of magnetization and electric polarization on the anomalous trans- port coefficients of a chiral fluid, New J. Phys. 19, 053014 (2017).

[59] The first attempt in this way, including CME however mostly numerically, was made in [60].

[60] S. F. Taghavi and U. A. Wiedemann, Chiral magnetic wave in an expanding QCD fluid, Phys. Rev. C 91, 024902 (2015).

[61] Y. Akamatsu, A. Mazeliauskas, and D. Teaney, A kinetic regime of hydrodynamic fluctuations and long time tails for a Bjorken expansion, Phys. Rev. C 95, 014909 (2017).

[62] N. Yamamoto, Chiral transport of neutrinos in supernova: Neutrino-induced fluid helicity and helical plasma instability, Phys. Rev. D 93, 065017 (2016). 